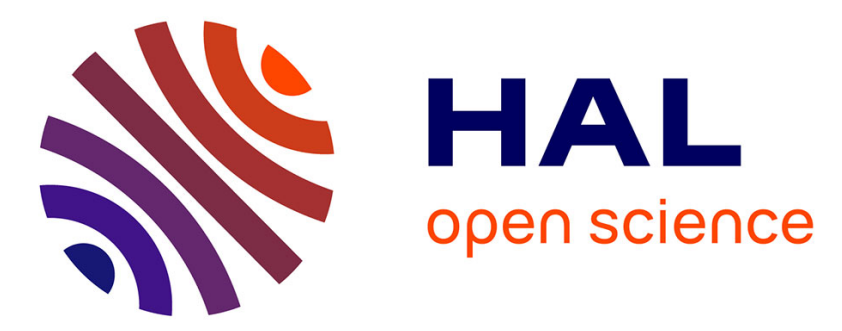

\title{
Long time existence results for Hamiltonian non-linear Klein-Gordon equations on some compact manifolds Jean-Marc Delort
}

\section{To cite this version:}

Jean-Marc Delort. Long time existence results for Hamiltonian non-linear Klein-Gordon equations on some compact manifolds. The Role of Metrics in the Theory of Partial Differential Equations. Proceedings of the 11th Mathematical Society of Japan, Seasonal Institute (MSJ-SI), Nagoya University, Japan, July 2-13 2018, Jul 2018, Sapporo, Japan. 10.2969/aspm/08510001 . hal-01892142

\section{HAL Id: hal-01892142 \\ https://hal.science/hal-01892142}

Submitted on 10 Oct 2018

HAL is a multi-disciplinary open access archive for the deposit and dissemination of scientific research documents, whether they are published or not. The documents may come from teaching and research institutions in France or abroad, or from public or private research centers.
L'archive ouverte pluridisciplinaire HAL, est destinée au dépôt et à la diffusion de documents scientifiques de niveau recherche, publiés ou non, émanant des établissements d'enseignement et de recherche français ou étrangers, des laboratoires publics ou privés. 


\title{
Long time existence results for Hamiltonian non-linear Klein-Gordon equations on some compact manifolds*
}

\author{
Jean-Marc Delort \\ Université Paris 13 , \\ Sorbonne Paris Cité, LAGA, CNRS (UMR 7539), \\ 99, Avenue J.-B. Clément, \\ F-93430 Villetaneuse
}

\begin{abstract}
Consider a nonlinear Klein-Gordon equation on $X$, a compact Riemannian manifold without boundary, $\left(\partial_{t}^{2}-\Delta+m^{2}\right) w=N(w)$, where $N$ is a smooth non-linearity. If the non-linearity vanishes at order $n+1$ at zero, and if the Cauchy data are of small size $\epsilon$ in a regular enough Sobolev space, the solution exists over time intervals of length $c / \epsilon^{n}$, and remains of size $O(\epsilon)$ over that interval of time.

In this review paper, we shall discuss the question of getting a similar existence result and $O(\epsilon)$ bounds over an interval of length larger than $c / \epsilon^{n}$. We shall present first some recent results concerning general manifolds, where one obtains some slight improvement of the lower bound of the time of existence given by local existence theory, when the mass parameter $m$ avoids a subset of zero measure.

Then, we turn to the question of obtaining lower bounds of the time of existence in $c_{N} \epsilon^{-N}$ for any $N$. It turns out that this is possible if one works on very special manifolds (Zoll manifolds), with Hamiltonian non linearities, and again takes the parameter $m$ of the equation outside a set of zero measure.

We shall focus first on the semi-linear case, where ideas of proof may be described easily. We shall then discuss with less details the more involved case of quasi-linear equations.
\end{abstract}

\section{Introduction}

The aim of this survey paper is to review results that have been obtained during the last ten years about long time existence for nonlinear hyperbolic equations with small smooth data on compact manifolds. We shall be interested mainly on nonlinear Klein-Gordon equations.

*Partially supported by the ANR project 13-BS01-0010-02 "Analyse asymptotique des équations aux dérivées partielles d'évolution". 
Denote by $(X, g)$ a compact Riemannian manifold of dimension $d \geq 1$, by $\Delta$ the Laplace-Beltrami operator on $X$. Let $m$ be given in $] 0,+\infty\left[\right.$ and let $n$ be in $\mathbb{N}^{*}$. We consider the following problem

$$
\begin{aligned}
\left(\partial_{t}^{2}-\Delta+m^{2}\right) w & =w^{n+1} \\
\left.w\right|_{t=0} & =\epsilon w_{0} \\
\left.\partial_{t} w\right|_{t=0} & =\epsilon w_{1}
\end{aligned}
$$

where $\epsilon>0$ is a small parameter and $\left(w_{0}, w_{1}\right)$ is in the unit ball of the product of Sobolev spaces $H^{s+1}(X, \mathbb{R}) \times H^{s}(X, \mathbb{R})$ for some large enough $s$. Our general goal is to obtain lower bounds for the length of the interval $]-T_{\epsilon}, T_{\epsilon}$ [ over which the solution exists and satisfies $\|w(t, \cdot)\|_{H^{s+1}}+\left\|\partial_{t} w(t, \cdot)\right\|_{H^{s}} \leq K \epsilon$ for some constant $K$.

Let us recall that a trivial lower bound for $T_{\epsilon}$, of the form $T_{\epsilon} \geq c \epsilon^{-n}$, is provided for free by local existence theory and the energy inequality associated to the equation. In fact, define the Sobolev energy

$$
E_{s}(w, t)=\|w(t, \cdot)\|_{H^{s+1}}^{2}+\left\|\partial_{t} u(t, \cdot)\right\|_{H^{s}}^{2}
$$

and consider the following statement:

There are constants $A_{0}>0, B_{0}>0, \epsilon_{0}>0$, such that, if $T \in\left[0, T_{\epsilon}[\right.$ and if the solution of (1.1) exists on $[0, T]$ and satisfies for any $t \in[0, T]$,

$$
\begin{aligned}
E_{s}(w, t) & \leq A_{0} \epsilon^{2} \\
\|w(t, \cdot)\|_{L^{\infty}} & \leq A_{1} \epsilon,
\end{aligned}
$$

then, actually, for $t$ in $[0, T]$ and $\epsilon \in] 0, \epsilon_{0}[$, one has

$$
\begin{aligned}
E_{s}(w, t) & \leq \frac{1}{2} A_{0} \epsilon^{2} \\
\|w(t, \cdot)\|_{L^{\infty}} & \leq \frac{1}{2} A_{1} \epsilon .
\end{aligned}
$$

Assume that the preceding statement has been proved for a value of $s$ which is large enough so that local existence and uniqueness holds for initial data in $H^{s+1} \times H^{s}$ (say $s>\frac{d}{2}$ ). Then the solution may be extended up to time $T_{\epsilon}$. Actually, one has just to consider the supremum $\tilde{T}_{\epsilon}$ of those $T \in\left[0, T_{\epsilon}[\right.$ such that the solution exists on $[0, T]$ and satisfies (1.3) on $[0, T]$. Then, we deduce from the implication $(1.3) \Rightarrow(1.4)$ that the solution of (1.1) satisfies (1.4) on $[0, T]$. By local existence theory, $w$ may be extended to some slightly larger interval $[0, T+\delta]$, and since the left hand side of (1.4) is a continuous function of time, if (1.4) holds on $[0, T]$, then (1.3) will be satisfied on $[0, T+\delta]$, for some $\delta$ independent of $T<\tilde{T}_{\epsilon}$. This shows that $\tilde{T}_{\epsilon}$ cannot be smaller than $T_{\epsilon}$.

In order to get lower bounds for $T_{\epsilon}$, we thus just have to establish $(1.3) \Rightarrow(1.4)$ for convenient values of this $T_{\epsilon}$. The trivial lower bound $T_{\epsilon} \geq c \epsilon^{-n}$ is obtained in that way: Computing the time derivative of the energy (1.2) and using the equation, we get for any $t \in\left[0, T_{\epsilon}[\right.$ the estimate

$$
E_{s}(w, t) \leq E_{s}(w, 0)+C \int_{0}^{t}\left\|w(\tau, \cdot)^{n+2}\right\|_{H^{s}} d \tau
$$


If we bound $\|w\|_{H^{s}}^{n+2} \leq C\|w\|_{L^{\infty}}^{n}\|w\|_{H^{s}}^{2}$ and use (1.3), we get

$$
E_{s}(w, t) \leq E_{s}(w, 0)+C t A_{1}^{n} A_{0} \epsilon^{n+2} .
$$

If $A_{0}$ has been chosen large enough relatively to the Cauchy data, we may assume that $E_{s}(w, 0) \leq \frac{A_{0}}{4} \epsilon^{2}$, so that, if we take $t \leq T_{\epsilon}=c \epsilon^{-n}$ with $c$ small enough, we obtain from (1.6) that the first inequality (1.4) holds true. The fact that the second equation (1.4) is satisfies follows just from Sobolev embedding (since $s>\frac{d}{2}$ ) and a convenient choice of $A_{1}$ in terms of $A_{0}$.

The question we want to address is the following one:

Can we extend the solution of (1.1), together with a bound of the form (1.3), over a longer time interval than $c \epsilon^{-n}$ ? More precisely, can we get for $T_{\epsilon}$ a lower bound $T_{\epsilon} \geq c \epsilon^{-A n}$ for some $A>1$ ? Under convenient assumptions, could we eventually get such a lower bound for any $A>1$ ?

Remark. Let us stress here the difference between the problem we consider and the corresponding one on $X=\mathbb{R}^{d}$, with small, smooth and decaying Cauchy data. In that framework, because of the dispersive properties of the equation, solutions are expected to decay in $L^{\infty}$ norm: $\|w(t, \cdot)\|_{L^{\infty}}=O\left(t^{-\frac{d}{2}}\right)$ when $t$ goes to infinity. This decay allows for much better lower bounds for the time of existence. For instance, in dimension larger or equal to three, one gets that for any $n \geq 1$, solutions of (1.1) with small smooth decaying initial data are global. The proof relies on bounds of the form (1.3), (1.4) with $A_{1}$ replaced by $A_{1} t^{-\frac{d}{2}}$. The new ingredient that is needed, in addition to energy inequality, is a way to exploit the dispersion in order to obtain the $L^{\infty}$ bounds in (1.3), (1.4). We refer to the papers of Klainerman [18] and Shatah [19] where this results are proved (including for more general non-linearities than those of (1.1)) and to the recent paper of Stingo [21] for up to date references on that dispersive case.

On the other hand, when $X$ is a compact manifold, we no longer have any dispersion, and one must rely on other properties in order to extend solutions beyond the time $c \epsilon^{-n}$ given by local existence theory. The key tool will be normal forms. We shall first describe this method on a simple example in next section.

\section{The normal forms method}

The normal forms method, that is very classical for ordinary differential equations, has been introduced for PDEs by Shatah [19] and Simon-Taflin [20]. To apply it to equation (1.1), let us introduce the following notation. Define by functional calculus $\Lambda_{m}=\sqrt{-\Delta+m^{2}}$, set $D_{t}=\frac{1}{i} \frac{\partial}{\partial t}$ and introduce

$$
w_{+}=\left(D_{t}+\Lambda_{m}\right) w, w_{-}=\left(D_{t}-\Lambda_{m}\right) w=-\bar{w}_{+}
$$

so that (1.1) may be written

$$
\left(D_{t}-\Lambda_{m}\right) w_{+}=F\left(w_{+}, w_{-}\right)
$$

with

$$
F\left(w_{+}, w_{-}\right)=\left(\frac{1}{2} \Lambda_{m}^{-1}\left(w_{+}-w_{-}\right)\right)^{n+1}
$$


Setting $u=w_{+}$, we see that the non-linearity in (2.1) may be written as a sum for $p=0, \ldots, n+1$ of expressions $M(u, \ldots, u, \bar{u}, \ldots, \bar{u})$ with $p$ arguments $u$ and $n+1-p$ arguments $\bar{u}, M\left(u_{1}, \ldots, u_{n+1}\right)$ being $(n+1)$-linear. Consider as a model the case of the non-linearity given by just one of these contributions, namely the equation

$$
\left(D_{t}-\Lambda_{m}\right) u=M(\underbrace{u, \ldots, u}_{p}, \underbrace{\bar{u}, \ldots, \bar{u}}_{n+1-p})
$$

for some $0 \leq p \leq n+1$. The basic idea of normal forms is to find another multilinear map $\tilde{M}\left(u_{1}, \ldots, u_{p+1}\right)$, chosen in such a way that if $v=u-\tilde{M}(u, \ldots, u, \bar{u}, \ldots, \bar{u})$, then

$$
\begin{gathered}
u \rightarrow v \text { is a local diffeomorphism at zero in } H^{s}(\text { for } s \gg 1), \\
v \text { solves an equation }\left(D_{t}-\Lambda_{m}\right) v=O\left(\|v\|_{H^{s}}^{n^{\prime}+1}\right)
\end{gathered}
$$

for some $n^{\prime}>n$. If (2.4), (2.5) hold, one will obtain an energy inequality

$$
\|v(t, \cdot)\|_{H^{s}}^{2} \leq\|v(0, \cdot)\|_{H^{s}}^{2}+C \int_{0}^{t}\|v(\tau, \cdot)\|_{H^{s}}^{n^{\prime}+2} d \tau
$$

so that, by the same reasoning as in the introduction, one will get that $v$ exists and stays of norm $O(\epsilon)$ in $H^{s}$ over an interval of length $c \epsilon^{-n^{\prime}}$. By (2.4), the solution $u$ of (2.3) satisfies as well such a bound. Since $n^{\prime}>n$, one has thus obtained an improved lower bound for the time of existence.

\section{The case of a quadratic non-linearity on the circle}

Let us explain how an $\tilde{M}$ such that (2.4), (2.5) hold may be constructed in the case when the non-linearity $M$ in (2.3) is quadratic and $X=\mathbb{S}^{1}$ the unit circle, following essentially Bourgain [6] (See also the papers of Bambusi [1] and BambusiGrébert [3]). For $k$ in $\mathbb{N}$, denote by $\Pi_{k}$ the spectral projector associated to the eigenvalue $k$ of $\sqrt{-\Delta}$ i.e. if $u$ is in $L^{2}\left(\mathbb{S}^{1}, \mathbb{C}\right)$, set

$$
\Pi_{0} u=\frac{\hat{u}(0)}{\sqrt{2 \pi}}, \Pi_{k} u=\hat{u}(k) \frac{e^{i k x}}{\sqrt{2 \pi}}+\hat{u}(-k) \frac{e^{-i k x}}{\sqrt{2 \pi}}
$$

where $\hat{u}(\xi)=\frac{1}{\sqrt{2 \pi}} \int_{-\pi}^{\pi} e^{-i x \xi} u(x) d x$ for $\xi$ in $\mathbb{Z}$. We have thus

$$
\mathrm{Id}=\sum_{k=0}^{+\infty} \Pi_{k} .
$$

Let $u$ be a solution of (2.3) and let us look for a multilinear expression

$$
\left(u_{1}, \ldots, u_{n+1}\right) \rightarrow \tilde{M}\left(u_{1}, \ldots, u_{n+1}\right)
$$

such that, if we set

$$
v=u-\tilde{M}(\underbrace{u, \ldots, u}_{p}, \underbrace{\bar{u}, \ldots, \bar{u}}_{n+1-p})
$$


we obtain for $v$ an equation of the form (2.5). Compute

$$
\begin{aligned}
\left(D_{t}-\Lambda_{m}\right) v & =\left(D_{t}-\Lambda_{m}\right) u-\sum_{j=1}^{p} \tilde{M}\left(u, \ldots, D_{t} u, \ldots, u, \bar{u}, \ldots, \bar{u}\right) \\
& +\sum_{j=p+1}^{n+1} \tilde{M}\left(u, \ldots, u, \bar{u}, \ldots, \overline{D_{t} u}, \ldots, \bar{u}\right)+\Lambda_{m} \tilde{M}(u, \ldots, u, \bar{u}, \ldots, \bar{u}) .
\end{aligned}
$$

Using equation (2.3), we may rewrite the right hand side of (2.11) as a term homogeneous of degree $n+1$, namely

$$
\begin{aligned}
& M(u, \ldots, u, \bar{u}, \ldots, \bar{u})-\sum_{j=1}^{p} \tilde{M}\left(u, \ldots, \Lambda_{m} u, \ldots, u, \bar{u}, \ldots, \bar{u}\right) \\
& \quad+\sum_{j=p+1}^{n+1} \tilde{M}\left(u, \ldots, u, \bar{u}, \ldots, \Lambda_{m} \bar{u}, \ldots, \bar{u}\right)+\Lambda_{m} \tilde{M}(u, \ldots, u, \bar{u}, \ldots, \bar{u})
\end{aligned}
$$

and contributions homogeneous of degree $n^{\prime}=2 n+1>n+1$, that will eventually be $O\left(\|u\|_{H^{s}}^{n^{\prime}+1}\right)$ in $H^{s}$ if we get good enough bounds on $\tilde{M}$. We thus have to find $\tilde{M}$ so that (2.12) vanishes, in other words, we need to solve the equation between $n+1$-linear maps

$$
\begin{aligned}
\sum_{j=1}^{p} \tilde{M}\left(u_{1}, \ldots, \Lambda_{m} u_{j}, \ldots, u_{n+1}\right) & -\sum_{j=p+1}^{n+1} \tilde{M}\left(u_{1}, \ldots, \Lambda_{m} u_{j}, \ldots, u_{n+1}\right) \\
& -\Lambda_{m} \tilde{M}\left(u_{1}, \ldots, u_{n+1}\right)=M\left(u_{1}, \ldots, u_{n+1}\right) .
\end{aligned}
$$

In (2.13), replace $u_{j}$ by $\Pi_{k_{j}} u_{j}$ for $j=1, \ldots, n+1$, where $k_{j}$ is in $\mathbb{N}$. Then, by definition of $\Pi_{k_{j}}, \Lambda_{m} \Pi_{k_{j}} u_{j}=\sqrt{m^{2}+k_{j}^{2}} \Pi_{k_{j}} u_{j}$, so that, if we make act also on the left on (2.13) another spectral projector $\Pi_{k_{n+2}}$, we reduce (2.13) to

$$
\mathcal{G}_{m}^{p}\left(k_{1}, \ldots, k_{n+2}\right) \Pi_{k_{n+2}} \tilde{M}\left(\Pi_{k_{1}} u_{1}, \ldots, \Pi_{k_{n+1}} u_{n+1}\right)=\Pi_{k_{n+2}} M\left(\Pi_{k_{1}} u_{1}, \ldots, \Pi_{k_{n+1}} u_{n+1}\right)
$$

where

$$
\mathcal{G}_{m}^{p}\left(k_{1}, \ldots, k_{n+2}\right)=\sum_{j=1}^{p} \sqrt{m^{2}+k_{j}^{2}}-\sum_{j=p+1}^{n+2} \sqrt{m^{2}+k_{j}^{2}} .
$$

The construction of $\tilde{M}$ is thus reduced to the division problem (2.14) and so to the fact that one may obtain from (2.15) lower bounds that are good enough so that (2.4) will hold true.

Let us consider first the case $n=1, p=2$, which corresponds to (2.3) with a quadratic non-linearity of the form $M(u, u)$. Notice also that by the definition $(2.7)$ of $\Pi_{k}$ and the fact that $e^{i k_{1} x} e^{i k_{2} x}=e^{i\left(k_{1}+k_{2}\right) x}$, both sides of (2.14) vanish identically unless for some choice of $\epsilon_{j} \in\{-1,1\}$

$$
\sum_{j=1}^{n+2} \epsilon_{j} k_{j}=0
$$


so that we have only to get lower bounds for (2.15) under the restriction (2.16). In the case $n=1, p=2$, we have

$$
\left|\mathcal{G}_{m}^{2}\left(k_{1}, k_{2}, k_{3}\right)\right|=\left|\sqrt{m^{2}+k_{1}^{2}}+\sqrt{m^{2}+k_{2}^{2}}-\sqrt{m^{2}+k_{3}^{2}}\right| \geq c\left(1+\min \left(k_{1}, k_{2}, k_{3}\right)\right)^{-1}
$$

under the restriction (2.16), as follows from elementary computations. Consequently, we may define $\tilde{M}$ from (2.14) dividing by $\mathcal{G}_{m}^{2}$, and we get a bound

$$
\left\|\Pi_{k_{3}} \tilde{M}\left(\Pi_{k_{1}} u_{1}, \Pi_{k_{2}} u_{2}\right)\right\|_{L^{2}} \leq C\left(1+\min \left(k_{1}, k_{2}, k_{3}\right)\right)\left\|\Pi_{k_{3}} M\left(\Pi_{k_{1}} u_{1}, \Pi_{k_{2}} u_{2}\right)\right\|_{L^{2}} .
$$

Assume that $M$ satisfies

$$
\left\|\Pi_{k_{3}} M\left(\Pi_{k_{1}} u_{1}, \Pi_{k_{2}} u_{2}\right)\right\|_{L^{2}} \leq C\left(1+\min \left(k_{1}, k_{2}, k_{3}\right)\right)^{\nu} \prod_{j=1}^{2}\left\|u_{j}\right\|_{L^{2}}
$$

for some $\nu$ (This is the case if $M\left(u_{1}, u_{2}\right)=u_{1} u_{2}$ for instance, with $\nu=0$ ). Then a similar estimate is satisfied by $\tilde{M}$ according to (2.18), with $\nu$ replaced by $\nu+1$, and one easily deduces from this and from condition (2.16) that $\tilde{M}$ is bounded from $H^{s} \times H^{s}$ to $H^{s}$, if $s$ is large enough relatively to $\nu$. It thus follows that (2.4), (2.5) hold with $n^{\prime}=2$ in the case of a quadratic non-linearity, so that an equation like (2.3) with right hand side $u^{2}$ and initial data of size $\epsilon$ in $H^{s}\left(\mathbb{S}^{1}\right)$, with $s$ large enough, has a solution defined on an interval of length at least $c \epsilon^{-2}$. The same holds for the non-linearities $\bar{u}^{2}$ and $|u|^{2}$.

We would like to obtain a similar improvement of the time of existence when the right hand side of (2.3) is homogeneous of degree $n+1$, with $n \geq 2$. We find immediately a difficulty: if $n \geq 2$, expression (2.15) for arguments $\left(k_{1}, \ldots, k_{n+2}\right) \in$ $\mathbb{N}^{n+2}$ satisfying (2.16) may vanish for some values of $m$. The idea is thus to exclude some values of $m$, i.e. to show that there is a subset $\mathcal{N}$ of $] 0,+\infty$ [ of zero measure such that, if $m$ is in $] 0,+\infty\left[-\mathcal{N}\right.$, there are $c_{0}>0, N_{0} \in \mathbb{N}$, such that

$$
\left|\mathcal{G}_{m}^{p}\left(k_{1}, \ldots, k_{n+2}\right)\right| \geq c\left(1+\max _{3}\left(k_{1}, \ldots, k_{n+2}\right)\right)^{-N_{0}}
$$

for any $\left(k_{1}, \ldots, k_{n+2}\right)$ satisfying $(2.16), \max _{3}\left(k_{1}, \ldots, k_{n+2}\right)$ being the third largest among these integers. If an inequality of the form (2.20) holds, one may repeat the above reasoning, and construct from (2.14) an $\tilde{M}$ such that (2.4), (2.5) are satisfied. One then extends the solution of (2.3) at least over a time interval of length $c \epsilon^{-2 n}$.

We shall see below that one may obtain an estimate of the form (2.20) when $n$ is odd (i.e. for non-linearities homogeneous of even degree) if $X=\mathbb{S}^{1}$ or more generally a Zoll manifold. On the other hand, if $n$ is even, there are some $k_{j}$ 's for which the left hand side of (2.20) vanishes identically (when in (2.15), $p=\frac{n}{2}+1$ and $\left.\left\{k_{1}, \ldots, k_{p}\right\}=\left\{k_{p+1}, \ldots, k_{n+2}\right\}\right)$. We shall have to identify then a special structure of the non-linearity that ensures that the corresponding contributions - that cannot be eliminated - do not make grow the Sobolev energy.

In the case of a general manifold, (2.20) does not hold true in general. Nevertheless, we shall see that in this case, a weaker property may be obtained. For equations like (1.1), this property will be sufficient to perform a (partial) normal form and obtain a slight improvement of the time of existence. We shall describe that in next section. 


\section{Semi-linear equations on a compact manifold}

We consider in this section $(X, g)$ a compact Riemannian manifold. We still denote by $-\Delta$ the positive Laplace operator on $X$. Our main result asserts that, for solutions of an equation of the form (1.1), we may find a lower bound for the time of existence in $c \epsilon^{-A n}$, for some constant $A>1$. More precisely, we have the following statement, obtained in joint work with Rafik Imekraz [11] (see also [8, 14] in the case of the torus):

Theorem 1 Let $(X, g)$ be a compact Riemannian manifold. There is $\mathcal{N} \subset] 0,+\infty[$, a subset of zero measure, and for any $m$ in $] 0,+\infty\left[-\mathcal{N}\right.$, there are $A>1, s_{0}>0$, and for any $s>s_{0}$, there are $c>0, K>0, \epsilon_{0}>0$, such that, for any $\left(w_{0}, w_{1}\right)$ in the unit ball for $H^{s+1}(X, \mathbb{R}) \times H^{s}(X, \mathbb{R})$, any $\left.\epsilon \in\right] 0, \epsilon_{0}\left[\right.$, equation (1.1) with data $\left.w\right|_{t=0}=\epsilon w_{0}$, $\left.\partial_{t} w\right|_{t=0}=\epsilon w_{1}$, has a unique solution $w$ in $C^{0}(]-T_{\epsilon}, T_{\epsilon}\left[, H^{s+1}\right) \cap C^{1}(]-T_{\epsilon}, T_{\epsilon}\left[, H^{s}\right)$ with $T_{\epsilon} \geq c \epsilon^{-A n}$. Moreover for any $\left.t \in\right]-T_{\epsilon}, T_{\epsilon}[$, one has

$$
\|w(t, \cdot)\|_{H^{s+1}}+\left\|\partial_{t} w(t, \cdot)\right\|_{H^{s}} \leq K \epsilon .
$$

The idea of proof will be to try to adapt the normal forms method to the general situation of compact manifolds. Several difficulties are in order. The first one is that we cannot expect to use a decomposition of the identity like (2.8), in terms of the spectral projectors associated to the individual eigenvalues. Actually, in order to get lower bounds for (2.15) (with $k_{j}$ replaced by some eigenvalue $\xi_{k_{j}}$ ), we need to have some lower estimate on the gap between consecutive distinct eigenvalues. But on a general manifold, one has no control on such a gap. Our idea will be to replace in (2.8) spectral projectors associated to one eigenvalue by spectral projectors on an interval of the spectrum, showing that we may obtain a convenient lower bound for the gap between two consecutive intervals. More precisely, we have the following proposition:

Proposition 2 Denote by $\left(\mu_{j}\right)_{j \in \mathbb{N}}$ the increasing sequence of distinct eigenvalues of $\sqrt{-\Delta}$ on $X$. There are a constant $\alpha \geq 1$ and a strictly increasing sequence of integers $\left(j_{k}\right)_{k \in \mathbb{N}}$, such that the following properties hold for the intervals $I_{0}=\left[0, \mu_{j_{0}}\right]$ and $I_{k}=\left[\mu_{j_{k-1}+1}, \mu_{j_{k}}\right](k \geq 1)$, when $k$ goes to infinity:

$$
\begin{aligned}
& \operatorname{dist}\left(I_{k}, 0\right)=\mu_{j_{k-1}+1}=3 \alpha k+O(1) \\
& \operatorname{Length}\left(I_{k}\right)=\mu_{j_{k}}-\mu_{j_{k-1}+1}=O(1) \\
& \operatorname{dist}\left(I_{k}, I_{k+1}\right)=\mu_{j_{k}+1}-\mu_{j_{k}} \geq c \mu_{j_{k}}^{-(d-1)} \sim c k^{-(d-1)} \\
& \operatorname{Card}\left(I_{k}\right) \cap \operatorname{Spect}(\sqrt{-\Delta})=O\left(k^{d-1}\right) .
\end{aligned}
$$

The geometric situation described by the above proposition may be illustrated by the following picture: 


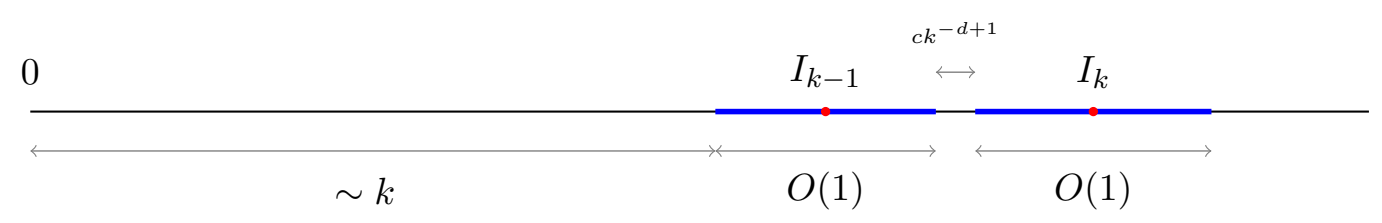

Figure 1: Spectrum of $\sqrt{-\Delta}$

We refer to [11] for the proof of Proposition 2. The fact that it holds on any compact manifold is a consequence of the Weyl formula, asserting that if $\left(\lambda_{j}\right)_{j}$ denotes the sequence of eigenvalues of $\sqrt{-\Delta}$ counted with multiplicities, then

$$
\operatorname{Card}\left\{j \in \mathbb{N} ; \lambda_{j} \leq \lambda\right\}=c(d) \operatorname{Vol}(X) \lambda^{d}+O\left(\lambda^{d-1}\right), \lambda \rightarrow+\infty,
$$

for some constant $c(d)$ depending only on the dimension. One deduces from that that there are constants $\alpha \geq 1, C>0$, such that, for any $k$ in $\mathbb{N}$

$$
\left.\left.C^{-1}(k+1)^{d-1} \leq \operatorname{Card}\left\{j \in \mathbb{N} ; \lambda_{j} \in\right] \alpha k, \alpha(k+1)\right]\right\} \leq C(k+1)^{d-1} .
$$

One then uses this to construct an increasing sequence $\left(j_{k}\right)_{k}$ of integers such that the eigenvalues $\left(\mu_{j}\right)_{j}$ of $\sqrt{-\Delta}$, counted without multiplicities, satisfy, for this subsequence of integers,

$$
3 \alpha k \leq \mu_{j_{k}} \leq 3 \alpha(k+1), \mu_{j_{k}+1}-\mu_{j_{k}} \geq \frac{1}{C \mu_{j_{k}}^{d-1}},
$$

which gives the proposition.

We define now

$$
\Pi_{k}=\mathbf{1}_{I_{k}}(\sqrt{-\Delta})
$$

so that $\mathrm{Id}=\sum_{k=0}^{+\infty} \Pi_{k}$. We would like to prove Theorem 1 using the same ideas as in section 2. We have seen there, in the case of equation (1.1) on the circle, that we could perform a normal forms method as soon as we have a lower bound on small divisors of the form (2.17) or (2.20). We would thus need here a control of a similar quantity. It turns out that, for a general compact manifold, we cannot expect as good a lower bound as in (2.20). We shall be able to obtain only the following weaker statement on small divisors.

Proposition 3 There is a subset $\mathcal{N}$ of $] 0,+\infty[$ and for every $m$ in $] 0,+\infty[-\mathcal{N}$, there are $c>0, N_{0} \in \mathbb{N}$, such that, for any $p \in[0, n+2]$, any $\left(k_{1}, \ldots, k_{n+2}\right) \in \mathbb{N}^{n+2}$, any $\left(\xi_{k_{1}}, \ldots, \xi_{k_{n+2}}\right) \in \prod_{j=1}^{n+2}\left(I_{k_{j}} \cap \operatorname{Spec} \sqrt{-\Delta}\right)$, one has

$$
\left|\sum_{j=1}^{p} \sqrt{m^{2}+\xi_{k_{j}}^{2}}-\sum_{j=p+1}^{n+2} \sqrt{m^{2}+\xi_{k_{j}}^{2}}\right| \geq c\left(1+\max \left(\xi_{k_{1}}, \ldots, \xi_{k_{n+2}}\right)\right)^{-N_{0}}
$$

except if $n$ is even, $p=\frac{n}{2}+1$ and

$$
\left\{k_{1}, \ldots, k_{p}\right\}=\left\{k_{p+1}, \ldots, k_{n+2}\right\} .
$$


The proof of the proposition follows from [12]. The idea is the following. For fixed $\left(k_{1}, \ldots, k_{n+2}\right)$ that do not satisfy $(3.5)$ when $n$ is even and $p=\frac{n}{2}+1$, the analytic function

$$
m \rightarrow \mathcal{G}_{m}^{p}\left(\xi_{k_{1}}, \ldots, \xi_{k_{n+2}}\right)=\sum_{j=1}^{p} \sqrt{m^{2}+\xi_{k_{j}}^{2}}-\sum_{j=p+1}^{n+2} \sqrt{m^{2}+\xi_{k_{j}}^{2}}
$$

is not identically zero. Consequently, it has on any compact interval $[a, b] \subset[0,+\infty[$ only finitely many zeros, and these zeros have bounded order. It follows (see next picture) that there are $\rho>0, C>0$, such that for any $\alpha \in] 0,1[$,

$$
\text { meas }\left\{m \in[a, b] ;\left|\mathcal{G}_{m}^{p}\left(\xi_{k_{1}}, \ldots, \xi_{k_{n+2}}\right)\right|<\alpha\right\} \leq C \alpha^{\rho} \text {. }
$$

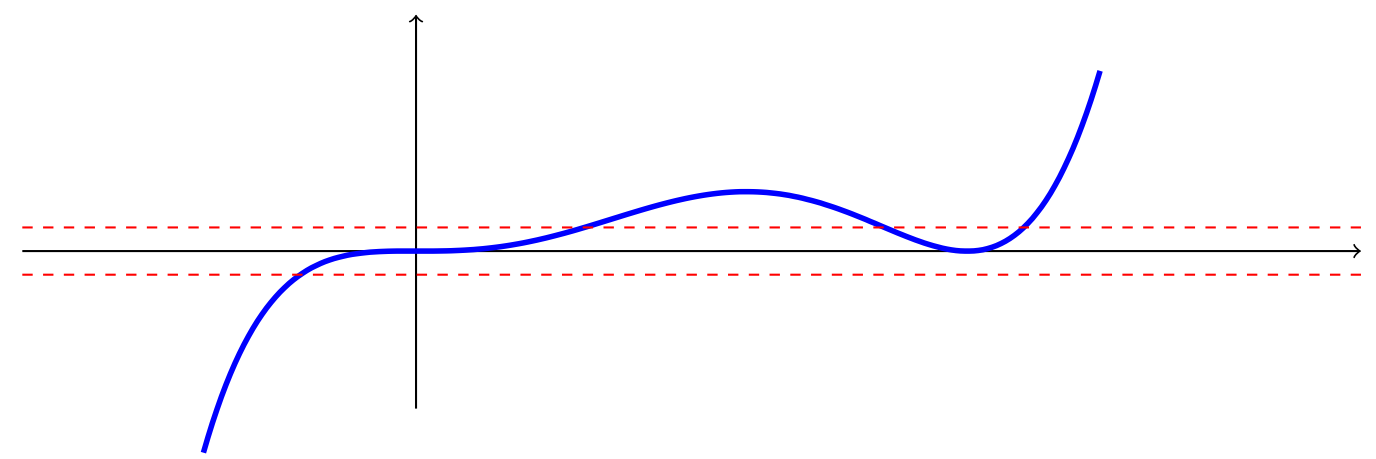

Of course, $C$ and $\rho$ in (3.7) may depend on the $k_{j}$ 's and the key of the proof of Proposition 3 is to show that $\rho$ may be taken independent of $\left(k_{1}, \ldots, k_{n+2}\right)$ and $C=O\left(\max \left(k_{1}, \ldots, k_{n+2}\right)^{N_{1}}\right)$ for some $N_{1}$. Taking $N_{0} \gg N_{1}$ and $\alpha$ of the form $\delta\left(1+\max \left(k_{1}, \ldots, k_{n+2}\right)\right)^{-N_{0}}$, one deduces from (3.7) that (3.4) holds true, except if $m$ is in a subset of measure $O\left(\delta^{\rho}\right)$.

Idea of proof of Theorem 1: Let us explain the idea of the proof on a model of the form (2.3):

$$
\left(D_{t}-\Lambda_{m}\right) u=\left(\Lambda_{m}^{-1} u\right)^{p}\left(\Lambda_{m}^{-1} \bar{u}\right)^{n+1-p}=M(\underbrace{\Lambda_{m}^{-1} u, \ldots, \Lambda_{m}^{-1} u}_{p}, \underbrace{\Lambda_{m}^{-1} \bar{u}, \ldots, \Lambda_{m}^{-1} \bar{u}}_{n+1-p}) .
$$

An important feature, that we shall use below, is the presence of the operator of degree $-1 \Lambda_{m}^{-1}$ acting on each factor in the right hand side of (3.8). Assume also to simplify the argument that $n$ is odd, and let us also pretend (which is of course not true in general!) that for any $k, I_{k} \cap \operatorname{Spec}(\sqrt{-\Delta})$ is reduced to a single (multiple) eigenvalue $\xi_{k}$. The general case may be obtained by a technical modification of the arguments we outline below. Define also the Sobolev norm on the manifold $X$ by

$$
\Theta_{s}^{0}(u)=\|u\|_{H^{s}}^{2}=\sum_{k=0}^{+\infty}(k+1)^{2 s}\left\|\Pi_{k} u\right\|_{L^{2}}^{2}
$$

with the spectral projectors $\Pi_{k}$ defined in (3.3). Using the equation, a direct computation shows that, for $s$ large enough,

$$
\frac{d}{d t} \Theta_{s}^{0}(u(t, \cdot))=O\left(\|u(t, \cdot)\|_{H^{s}}^{n+2}\right),
$$


so that one gets an estimate of the form (1.5) for $t \geq 0$ :

$$
\Theta_{s}^{0}(u(t, \cdot)) \leq \Theta_{s}^{0}(u(0, \cdot))+C \int_{0}^{t}\|u(\tau, \cdot)\|_{H^{s}}^{n} \Theta_{s}^{0}(u(\tau, \cdot)) d \tau .
$$

This implies, as in (1.6), that a bound $\Theta_{s}^{0}(u(t, \cdot))=O\left(\epsilon^{2}\right)$ holds true over an interval of the form $\left[0, c \epsilon^{-n}\right]$, if the initial data is of size $\epsilon$ in $H^{s}$. In order to prove the theorem, we need to improve the power $n$ in the integral in the right hand side of (3.11) to $A n$ for some $A>1$. Let us notice first that in estimate (3.10), we did not use the presence of order -1 operators in the right hand side of (3.8). Actually, we have

$$
\frac{d}{d t} \Theta_{s}^{0}(u(t, \cdot))=2 \operatorname{Re}\left\langle u, M\left(\Lambda_{m}^{-1} u, \ldots, \Lambda_{m}^{-1} \bar{u}\right)\right\rangle_{H^{s}} .
$$

In the right hand side of (3.12), let us decompose

$$
u=u_{L}+u_{H}, u_{L}=\sum_{k ; k<\epsilon^{-\delta}} \Pi_{k} u
$$

for some small $\delta>0$ to be chosen. Consider first the contribution to the right hand side of (3.12) of $M\left(\Lambda_{m}^{-1} u_{1}, \ldots, \Lambda_{m}^{-1} u_{n+1}\right)$ where at least one of the $u_{j}$ 's has been replaced by $u_{H}$. Since

$$
\left\|\Lambda_{m}^{-1} u_{H}\right\|_{H^{s}} \leq C \epsilon^{\delta}\|u\|_{H^{s}}
$$

we shall get from (3.12)

$$
\frac{d}{d t} \Theta_{s}^{0}(u(t, \cdot))=2 \operatorname{Re}\left\langle u, M\left(\Lambda_{m}^{-1} u_{L}, \ldots, \Lambda_{m}^{-1} \bar{u}_{L}\right)\right\rangle_{H^{s}}+O\left(\epsilon^{\delta}\|u\|_{H^{s}}^{n+2}\right)
$$

and since $\epsilon$ is the size of the initial data, the last term in (3.15) is essentially of the form $O\left(\|u\|_{H^{s}}^{n^{\prime}+2}\right)$ with $n^{\prime}=n+\delta>n$. Consequently, if we ignore the first term in the right hand side of (3.15), we would get a bound of the form (3.11) with the power $n$ replaced by $n^{\prime}$, whence a lower estimate for the time of existence in $c \epsilon^{-n^{\prime}}$. In order to get the conclusion of the theorem, we have thus to show that we may get rid of the first term in the right hand side of (3.15) by normal forms. To do so, we shall look for a perturbation $\tilde{\Theta}_{s}(u)$ of $\Theta_{s}^{0}(u)$ such that

$$
\Theta_{s}^{0}(u)-\tilde{\Theta}_{s}(u) \sim \Theta_{s}^{0}(u) \text { for small } u
$$

and

$$
\frac{d}{d t}\left(\Theta_{s}^{0}(u(t, \cdot))-\tilde{\Theta}_{s}(u(t, \cdot))=O\left(\epsilon^{\delta^{\prime}}\|u(\tau, \cdot)\|^{n+2}\right)\right.
$$

for some $\delta^{\prime}>0$. If we may achieve (3.16) and (3.17), we shall get by integration for $t \geq 0$

$$
\begin{aligned}
& \|u(t, \cdot)\|_{H^{s}}^{2}=\Theta_{s}^{0}(u(t, \cdot)) \sim \Theta_{s}^{0}(u(t, \cdot))-\tilde{\Theta}_{s}(u(t, \cdot)) \\
& \quad \leq C\left[\|u(0, \cdot)\|_{H^{s}}^{2}+\epsilon^{\delta^{\prime}} \int_{0}^{t}\|u(\tau, \cdot)\|_{H^{s}}^{n+2} d \tau\right]
\end{aligned}
$$


which implies existence of the solution up to time of magnitude $\epsilon^{-\left(n+\delta^{\prime}\right)}$ and the conclusion.

In view of (3.15), property (3.17) will hold true if we construct $\tilde{\Theta}_{s}$ so that

$$
\frac{d}{d t} \tilde{\Theta}_{s}(u(t, \cdot))=2 \operatorname{Re}\left\langle u, M\left(\Lambda_{m}^{-1} u_{L}, \ldots, \Lambda_{m}^{-1} \bar{u}_{L}\right)\right\rangle_{H^{s}}+O\left(\epsilon^{\delta^{\prime}}\|u\|_{H^{s}}^{n+2}\right) .
$$

We look for $\tilde{\Theta}_{s}$ as

$$
\begin{aligned}
\tilde{\Theta}_{s}(u)=-2 \operatorname{Re} \sum_{k_{1}} \cdots \sum_{k_{n+2}}( & \left.k_{n+2}+1\right)^{2 s} i \mathcal{G}_{m}^{p}\left(\xi_{k_{1}}, \ldots, \xi_{k_{n+2}}\right)^{-1} \\
& \times \int \Pi_{k_{n+2}} \bar{u} M\left(\Lambda_{m}^{-1} \Pi_{k_{1}} u_{L}, \ldots, \Lambda_{m}^{-1} \Pi_{k_{n+1}} \bar{u}_{L}\right) d x,
\end{aligned}
$$

where $\xi_{k_{j}}$ is the unique point in $I_{k_{j}} \cap \operatorname{Spec}(\sqrt{-\Delta})$ and $\mathcal{G}_{m}^{p}$ is given by (3.6). We notice first that (3.16) will hold true if $\delta$ in (3.13) is chosen small enough. Actually, by (3.4) and the fact that all frequencies $\xi_{k_{j}}$ are smaller than $\epsilon^{-\delta}$ because of the definition of $u_{L},\left|\left(\mathcal{G}_{m}^{p}\right)^{-1}\right| \leq C \epsilon^{-\delta N_{0}}$, so that $\tilde{\Theta}_{s}(u)=O\left(\epsilon^{-\delta N_{0}}\|u\|_{H^{s}}^{n+2}\right)=O\left(\epsilon^{n-\delta N_{0}} \Theta_{s}^{0}(u)\right)$ as long as $\|u\|_{H^{s}}=O(\epsilon)$. If $\delta N_{0} \ll n$, we do get a small perturbation to $\Theta_{s}^{0}(u)$.

On the other hand, when we compute $\frac{d}{d t} \tilde{\Theta}_{s}(u(t, \cdot))$ and make act the time derivative on each argument in the right hand side of (3.20), we get using equation (3.8):

- Terms where $\frac{d u}{d t}$ is replaced by $i \Lambda_{m} u$. As $\Lambda_{m} \Pi_{k_{j}}=\sqrt{m^{2}+\xi_{k_{j}}^{2}} \Pi_{k_{j}}$ we obtain, because of the definition of $\mathcal{G}_{m}^{p}$, a contribution equal to the first term in the right hand side of (3.19).

- Terms where $\frac{d u}{d t}$ is replaced by the non-linearity in (3.8). These terms are bounded, using that $\left|\mathcal{G}_{m}^{p}\right|^{-1} \leq C \epsilon^{-\delta N_{0}}$ and that $\|u\|_{H^{s}}=O(\epsilon)$, by

$$
C\|u\|_{H^{s}}^{2 n+2} \epsilon^{-\delta N_{0}}=O\left(\epsilon^{n-\delta N_{0}}\|u\|_{H^{s}}^{n+2}\right),
$$

so that they contribute to the remainder in (3.19), with $\delta^{\prime}=n-\delta N_{0}>0$, if $\delta$ is small enough. This concludes the proof.

Remarks: - The proof we outlined above works for $n$ odd. When $n$ is even and $p=\frac{n}{2}+1$, the lower bound (3.4) does not hold for indices such that $\left\{k_{1}, \ldots, k_{p}\right\}=$ $\left\{k_{p+1}, \ldots, k_{n+2}\right\}$. One has then to check that these modes give a zero contribution when one computes (3.15). We shall come back to this issue in next section.

- Clearly, the proof of Theorem 1 uses in an essential way the fact that in equation (1.1), the non-linearity does not contain derivatives of $w$. This is what makes appear the operator $\Lambda_{m}^{-1}$ in the non-linearity of (3.8), these operators playing a seminal role in the proof of Theorem 1. In particular, the proof does not extend to non-linearities of the form $\left(\partial_{x} u\right)^{n+1}$ and even less to quasi-linear non-linearities, like $u^{n}\left(\partial_{x}^{2} u\right)$.

Nevertheless, let us mention a very recent result of Ionescu and Pusateri [16] of long time existence for solutions of capillary-gravity wave equations on $\mathbb{T}^{2}$, for almost all values of the gravity-surface tension parameters. This equations is quasilinear (actually even fully nonlinear). Though, it is possible to apply a normal form procedure in order to obtain a lower bound for the time of existence in $\epsilon^{-\frac{5}{3}}$, instead 
of the $\epsilon^{-1}$ that would be given by local existence theory. The key point is that the structure of the non-linearity allows one to gain derivatives, when eliminating quadratic contributions to the non-linearity that are spectrally localized in phase space regions giving rise to small divisors.

\section{Almost global existence on Zoll manifolds}

In the preceding section, Theorem 1 provided, for an equation of the form (1.1), a slight improvement of the time of existence of solutions with data of size $\epsilon$, through a normal forms method that was replacing a non-linearity vanishing at some order at zero by another one, vanishing at an higher order. A natural idea, in order to get a better improvement of the time of existence, and eventually reaching "almost global existence", i.e. existence over a time interval of length $c_{N} \epsilon^{-N}$ for any $N$, would be to iterate the normal forms construction, in order to reduce oneself to higher and higher order vanishing non-linearities. Clearly, one cannot expect to do so in the general framework of section 3: we remarked that we used at the first normal forms step the fact that the non-linearity in equation (3.8) involves the action of operators of degree -1 on each factor, and this property is lost on the higher order contributions generated by the reduction. Nevertheless, in dimension one, Bourgain [6] constructed iterated normal forms that allow one to get almost global existence of solutions. Our goal here is to obtain similar results in higher dimensions. We shall be able to do so only on a very restricted class of compact manifolds. Actually, in one space dimension, one uses several special facts: first, the property that the product of two eigenfunctions of the Laplace operator on the circle is still an eigenfunction: $e^{i k x} e^{i k^{\prime} x}=e^{i\left(k+k^{\prime}\right) x}$. It turns out that this may be replaced by an inequality that holds true on any compact manifold, so that this property is not essential for the proof of long time existence. Second, one uses an estimate of the form (2.20) that gives a control of small divisors in terms of a negative power of the third largest of their arguments. On a general manifold, we have only the lower bound (3.4), that involves a negative power of the largest frequency. This bound would provoke losses of derivatives in a normal forms procedure, and thus prevents one from using the method in general. We shall thus limit ourselves to the case of manifolds whose spectrum has special separation properties, so that a lower bound of type (2.20) will hold true. This is the class of Zoll manifolds, whose definition is recalled below.

Definition 4 A Zoll manifold is a compact Riemannian manifold $(X, g)$ on which the geodesic flow is periodic.

Spheres are obviously Zoll manifolds, but that class contains other examples (see Besse [5]). For our purposes, the key point will be a characterization of Zoll manifolds in terms of their spectral properties due to Weinstein [22], Colin de Verdière [7] and Guillemin [15].

Theorem 5 Let $X$ be a compact Riemannian manifold. There is equivalence between:

(i) $X$ is Zoll. 
(ii) There are $\tau>0$ (the period of the geodesic flow), $\delta>0, c_{0}>0, \alpha \in \mathbb{R}$ such that $\operatorname{Spec}(\sqrt{-\Delta}) \subset \bigcup_{n=0}^{+\infty} I_{k}$, where the union is disjoint, $I_{0}$ is some compact interval and for $k \geq 1$,

$$
I_{k}=\left[\frac{2 \pi}{\tau} k+\alpha-\frac{c_{0}}{k^{\delta}}, \frac{2 \pi}{\tau} k+\alpha+\frac{c_{0}}{k^{\delta}}\right] .
$$

Let us illustrate statement (ii) on a picture:

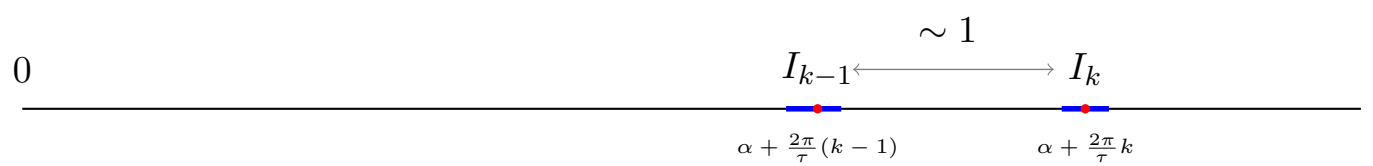

Figure 2: Spectrum of $\sqrt{-\Delta}$ on a Zoll manifold

The spectrum of $\sqrt{-\Delta}$ is contained in the union of intervals, centered at an arithmetic progression, whose length goes to zero as the energy increases, so that two consecutive $I_{k}$ 's are separated by a gap bounded from below by a constant. Notice that this is in sharp contrast with the situation described by the figure on page 8 , for a general manifold, where we could just include the spectrum in intervals separated by a shrinking gap. We may exploit this separation property in order to prove the following:

Proposition 6 Assume $X$ is Zoll. There is $\mathcal{N} \subset] 0,+\infty[$ of zero measure such that, for any $n \in \mathbb{N}^{*}$, any $\left.m \in\right] 0,+\infty\left[-\mathcal{N}\right.$, there are $c>0, N_{0} \in \mathbb{N}$, so that, for any $k_{1}, \ldots, k_{n+2}$ in $\mathbb{N}$, for any $\xi_{k_{j}} \in \operatorname{Spec}(\sqrt{-\Delta}) \cap I_{k_{j}}$, any $0 \leq p \leq n+2$, one has, using notation (3.6)

$$
\left|\mathcal{G}_{m}^{p}\left(\xi_{k_{1}}, \ldots, \xi_{k_{n+2}}\right)\right| \geq c\left(1+\max _{3}\left(k_{1}, \ldots, k_{n+2}\right)\right)^{-N_{0}}
$$

$\left(\max _{3}\left(k_{1}, \ldots, k_{n+2}\right)\right.$ standing for the third largest among $\left.\left(k_{1}, \ldots, k_{n+2}\right)\right)$, unless

$$
n \text { is even, } p=\frac{n+2}{2} \text { and }\left\{k_{1}, \ldots, k_{p}\right\}=\left\{k_{p+1}, \ldots, k_{n+2}\right\} \text {. }
$$

Idea of proof: By Proposition 3, we know that a lower bound for the left hand side of (4.2) in terms of $c\left(1+\max \left(k_{1}, \ldots, k_{n+2}\right)\right)^{-N_{0}}$ holds true, since $\xi_{k_{j}} \sim k_{j}$ for any $\xi_{k_{j}}$ in interval $I_{k_{j}}$. To get the better lower bound (4.2), let us assume for instance that $k_{1}, k_{n+2} \gg \max \left(k_{2}, \ldots, k_{n+1}\right)$ and write (when $1<p<n+2$, which is the only serious case),

$$
\mathcal{G}_{m}^{p}\left(\xi_{k_{1}}, \ldots, \xi_{k_{n+2}}\right)=\sqrt{m^{2}+\xi_{k_{1}}^{2}}-\sqrt{m^{2}+\xi_{k_{n+2}}^{2}}+\tilde{\mathcal{G}}_{m}^{p}\left(\xi_{k_{2}}, \ldots, \xi_{k_{n+1}}\right)
$$

with

$$
\tilde{\mathcal{G}}_{m}^{p}\left(\xi_{k_{2}}, \ldots, \xi_{k_{n+1}}\right)=\sum_{j=2}^{p} \sqrt{m^{2}+\xi_{k_{j}}^{2}}-\sum_{j=p+1}^{n+1} \sqrt{m^{2}+\xi_{k_{j}}^{2}} .
$$

Since $\xi_{k_{j}} \in I_{k_{j}}$, interval centered at $\alpha+\frac{2 \pi}{\tau} k_{j}$ according to (4.1), and since the length of these intervals is $O\left(k_{j}^{-\delta}\right)$, we may write

$$
\sqrt{m^{2}+\xi_{k_{1}}^{2}}-\sqrt{m^{2}+\xi_{k_{n+2}}^{2}}=\left(\alpha+\frac{2 \pi}{\tau} k_{1}\right)-\left(\alpha+\frac{2 \pi}{\tau} k_{n+2}\right)+O\left(k_{1}^{-\delta}\right)+O\left(k_{n+2}^{-\delta}\right)
$$


from which we can conclude

$$
\mathcal{G}_{m}^{p}\left(\xi_{k_{1}}, \ldots, \xi_{k_{n+2}}\right)=\frac{2 \pi}{\tau} q+\tilde{\mathcal{G}}_{m}^{p}\left(\xi_{k_{2}}, \ldots, \xi_{k_{n+1}}\right)+O\left(k_{1}^{-\delta}\right)+O\left(k_{n+2}^{-\delta}\right)
$$

where $q$ belongs to $\mathbb{Z}$. Because of that, and of the fact that the arguments of $\tilde{\mathcal{G}}_{m}^{p}$ are all $O\left(\max _{3}\left(k_{1}, \ldots, k_{n+2}\right)\right)$, a modification of the proof of Proposition 3 shows that, for any $q$ in $\mathbb{Z}$, any $\xi_{k_{j}} \in \operatorname{Spec}(\sqrt{-\Delta}) \cap I_{k_{j}}$, any $m$ outside a subset $\mathcal{N}$ of zero measure,

$$
\left|\frac{2 \pi}{\tau} q+\tilde{\mathcal{G}}_{m}^{p}\left(\xi_{k_{2}}, \ldots, \xi_{k_{n+1}}\right)\right| \geq c\left(1+\max _{3}\left(k_{1}, \ldots, k_{n+2}\right)\right)^{-N_{0}}
$$

for some $N_{0}$. As we may assume that

$$
k_{1}, k_{n+2} \geq \max _{3}\left(k_{1}, \ldots, k_{n+2}\right)^{N_{1}}
$$

for a large enough $N_{1}$ (since otherwise (4.2) follows from (3.4)), one sees that the perturbations of the right hand side of (4.7) are small enough so that (4.8) implies $(4.2)$.

According to Proposition 6, we have an improved bound (4.2) for small divisors in the case of Zoll manifolds. This will be the key point that will allow us to prove the following theorem due to Bambusi, Delort, Grébert and Szeftel [2]:

Theorem 7 Let $X$ be a Zoll manifold. Let $f:(x, w) \rightarrow f(x, w)$ from $X \times \mathbb{R}$ to $\mathbb{R}$ be smooth, vanishing at order three at $w=0$. There is $\mathcal{N} \subset] 0,+\infty[$, of zero measure and, for any $m \in] 0,+\infty\left[-\mathcal{N}\right.$, any $N \in \mathbb{N}$, there is $s_{0}>0$ and for any $s>s_{0}$, there are $\epsilon_{0}>0, c>0$, such that, for all $\left.\epsilon \in\right] 0, \epsilon_{0}\left[\right.$, any $\left(w_{0}, w_{1}\right)$ in the unit ball of $H^{s+\frac{1}{2}}(X, \mathbb{R}) \times H^{s-\frac{1}{2}}(X, \mathbb{R})$,

$$
\begin{aligned}
\left(\partial_{t}^{2}-\Delta+m^{2}\right) w & =\partial_{w} f(x, w) \\
\left.w\right|_{t=0} & =\epsilon w_{0} \\
\left.\partial_{t} w\right|_{t=0} & =\epsilon w_{1}
\end{aligned}
$$

has a unique solution

$$
w \in C^{0}(]-T_{\epsilon}, T_{\epsilon}\left[, H^{s+\frac{1}{2}}(X, \mathbb{R})\right) \cap C^{1}(]-T_{\epsilon}, T_{\epsilon}\left[, H^{s-\frac{1}{2}}(X, \mathbb{R})\right)
$$

with $T_{\epsilon} \geq c \epsilon^{-N}$. Moreover $\left(w(t, \cdot), \partial_{t} w(t, \cdot)\right)$ is $O(\epsilon)$ in $H^{s+\frac{1}{2}}(X, \mathbb{R}) \times H^{s-\frac{1}{2}}(X, \mathbb{R})$ for $t$ in $]-T_{\epsilon}, T_{\epsilon}[$.

The idea of proof of the above theorem will be to apply a sequence of normal forms, in order to reduce the non-linearity to terms of higher and higher order of homogeneity. It turns out that it is not possible to eliminate all contributions of lower degree: this is related to the fact that the small divisors estimate (4.2) can be achieved except when (4.3) holds. One has thus to check that the corresponding terms in the non-linearity do not make grow the Sobolev energy. For the first step in a normal forms procedure, this may be verified by hand. But along the iteration, the new nonlinear contributions induced by successive reductions become more and more complicated, so that the cancellation properties cannot be checked directly. 
Though, a way of achieving that in a systematic way is to put the problem in an Hamiltonian framework, in order to be able to use a Birkhoff normal forms method. Then terms corresponding to indices satisfying (4.3) are those contributions to the Hamiltonian that depend only on the actions, so that the fact that they do not make grow the energy becomes trivial. We introduce this Hamiltonian formulation in next section.

\section{Hamiltonian formulation and Birkhoff normal forms}

Denote by $\left\langle V, V^{\prime}\right\rangle=\int_{X} V \cdot V^{\prime} d x$ the usual scalar product on $L^{2}\left(X, \mathbb{R}^{2}\right.$ ) (where $d x$ stands for the Riemannian volume element). Define a symplectic form by

$$
\omega\left(V, V^{\prime}\right)=\left\langle{ }^{t} J V, V^{\prime}\right\rangle
$$

where $J=\left[\begin{array}{cc}0 & -1 \\ 1 & 0\end{array}\right]$. If $V \rightarrow G(V)$ is a $C^{1}$ function on an open subset of $H^{s}\left(X, \mathbb{R}^{2}\right)$, for some $s$, we denote by $\nabla G(V)$ the unique element of $H^{-s}\left(X, \mathbb{R}^{2}\right)$ such that, for any vector $H$ in $H^{s}\left(X, \mathbb{R}^{2}\right)$,

$$
D G(V) \cdot H=\langle\nabla G(V), H\rangle
$$

and the symplectic gradient $X_{G}(V)$ by

$$
X_{G}(V)=J \nabla G(V) .
$$

In the sequel, we shall always take $s$ large enough and consider only functions $G$ such that when $V$ is in $H^{s}$, then $\nabla G(V)$ or $X_{G}(V)$ belongs to $H^{s}$ (or $H^{s-1}$ ), like polynomials in $V$ for instance. The Hamiltonian equation associated to $G$ is

$$
\begin{aligned}
\partial_{t} V & =X_{G}(V) \\
\left.V\right|_{t=0} & =V_{0} \in H^{s}\left(X, \mathbb{R}^{2}\right) .
\end{aligned}
$$

Let us write the Klein-Gordon equation (4.9) under the form (5.3). Recall that we set

$$
\Lambda_{m}=\sqrt{-\Delta+m^{2}}
$$

and if we are given a function of $t$ in some interval $[0, T]$, with values in $H^{s+\frac{1}{2}}$, such that

$$
w \in C^{0}\left([0, T], H^{s+\frac{1}{2}}(X, \mathbb{R})\right) \cap C^{1}\left([0, T], H^{s-\frac{1}{2}}(X, \mathbb{R})\right),
$$

define

$$
V=\left[\begin{array}{c}
V^{1} \\
V^{2}
\end{array}\right]=\left[\begin{array}{c}
\Lambda_{m}^{-\frac{1}{2}} \partial_{t} w \\
\Lambda_{m}^{\frac{1}{2}} w
\end{array}\right] \in C^{0}\left([0, T], H^{s}\left(X, \mathbb{R}^{2}\right)\right)
$$

Let $(x, w) \rightarrow f(x, w)$ be a smooth function on $X \times \mathbb{R}$ with values in $\mathbb{R}$. Define

$$
F(w)=\int_{X} f(x, w(x)) d x
$$


This is a smooth function on $H^{s}(X, \mathbb{R})$ for $s$ large enough, such that $\nabla F(w)=$ $\frac{\partial f}{\partial w}(x, w(x))$ belongs to $H^{s}(X, \mathbb{R})$. Introduce

$$
G_{0}(V)=\frac{1}{2} \int_{X} V \cdot\left(\Lambda_{m} V\right) d x, G(V)=G_{0}(V)-F\left(\Lambda_{m}^{-\frac{1}{2}} V^{2}\right)
$$

using notation (5.4). Then the linear Klein-Gordon equation $\left(\partial_{t}^{2}-\Delta+m^{2}\right) w=0$ is equivalent to $\partial_{t} V=X_{G_{0}}(V)$ and equation (4.9) is equivalent to $\partial_{t} V=X_{G}(V)$. For simplification, assume that $f$ is a polynomial in $w$ independent of $x$. Then our system (4.9) may be written

$$
\begin{aligned}
\partial_{t} V & =X_{G}(V) \\
\left.V\right|_{t=0} & =\epsilon V_{0}
\end{aligned}
$$

with $V_{0}$ given in the unit ball of $H^{s}\left(X, \mathbb{R}^{2}\right)$ and $G(V)$ a polynomial $G(V)=\sum_{n=0}^{N^{\prime}} G_{n}(V)$, with

$$
\begin{aligned}
& G_{0}(V)=\frac{1}{2} \int_{X}\left(\Lambda_{m}^{\frac{1}{2}} V\right) \cdot\left(\Lambda_{m}^{\frac{1}{2}} V\right) d x \\
& G_{n}(V)=\int_{X} P_{n}\left(\Lambda_{m}^{-\frac{1}{2}} V\right) d x
\end{aligned}
$$

where $P_{n}$ is an homogeneous polynomial of order $n+2$.

Let us rewrite equation (5.7) in complex coordinates, introducing

$$
u=\frac{\sqrt{2}}{2}\left(V^{1}+i V^{2}\right)
$$

and

$$
\nabla_{u}=\frac{\sqrt{2}}{2}\left(\nabla_{V^{1}}-i \nabla_{V^{2}}\right), \nabla_{\bar{u}}=\frac{\sqrt{2}}{2}\left(\nabla_{V^{1}}+i \nabla_{V^{2}}\right)
$$

Then equation (5.7) may be rewritten in terms of $u$ as

$$
\begin{aligned}
\partial_{t} u & =i \nabla_{\bar{u}} G(u, \bar{u}) \\
\left.u\right|_{t=0} & =\epsilon u_{0}
\end{aligned}
$$

with $u_{0} \in H^{s}(X, \mathbb{C})$ and where, if we set $U=(u, \bar{u})$, the Hamiltonian $G$ may be rewritten from (5.8) as

$$
G=\sum_{n=0}^{N^{\prime}} G_{n}(U),
$$

with

$$
\begin{aligned}
G_{0}(U) & =\int_{X}\left(\Lambda_{m} u\right) \bar{u} d x \\
G_{n}(U) & =\sum_{p=0}^{n+2} \int_{X} P_{n, p}(u, \ldots, u, \bar{u}, \ldots, \bar{u})
\end{aligned}
$$


where $P_{n, p}$ is a $(n+2)$-linear expression, depending on $p$ arguments $u$ and $n+2-p$ arguments $\bar{u}$. We did not make appear in the expression of $P_{n, p}$ the operators $\Lambda_{m}^{-\frac{1}{2}}$ as they will play no role.

The strategy of proof of Theorem 7 will be the same as the one described in above sections 1 and 2, except that we shall iterate the normal forms construction, and will rely for that on the Hamiltonian framework. Denote

$$
\Theta_{s}^{0}(U)=\frac{1}{2} \int_{X}\left(\Lambda_{m}^{s} u\right)\left(\overline{\Lambda_{m}^{s} u}\right) d x \sim\|u\|_{H^{s}}^{2}
$$

the Sobolev energy. We shall take $s$ large enough and look for another function $\Theta_{s}(U)$ that satisfies

$$
\Theta_{s}(U) \sim\|u\|_{H^{s}}^{2} \text { if }\|u\|_{H^{s}} \ll 1
$$

and

$$
\frac{d}{d t} \Theta_{s}(U(t, \cdot))=O\left(\|u(t, \cdot)\|_{H^{s}}^{N+2}\right), U \rightarrow 0
$$

if $U$ is a solution of (5.11), and $N$ is an arbitrary given integer. As already seen, (5.15) and (5.16) imply that the solution may be extended up to a time in $c \epsilon^{-N}$, and that the Sobolev norm of the solution remains of size $O(\epsilon)$ over such an interval.

\section{Birkhoff normal forms method}

We look for $\Theta_{s}$ as

$$
\Theta_{s}(U)=\Theta_{s}^{0} \circ \chi(U)
$$

where $\chi$ is a local diffeomorphism from a neighborhood of zero in $H^{s}\left(X, \mathbb{R}^{2}\right) \simeq$ $H^{s}(X, \mathbb{C})$ to itself, sending zero to zero, that is assumed moreover symplectic. For $F, G$ two smooth maps on an open set of $H^{s}$, we define their Poisson bracket by

$$
\{F, G\}(U)=D G(U) \cdot X_{F}(U)
$$

(Assuming that $X_{F}(U)$ is sufficiently regular so that the action of $D G(U)$ on it makes sense, which will be the case in our applications below). In complex coordinates, one gets

$$
\{F, G\}(U)=i\left[D_{u} G(U) \cdot \nabla_{\bar{u}} F(U)-D_{\bar{u}} G(U) \cdot \nabla_{u} F(U)\right] .
$$

Moreover, if $\chi$ is symplectic, we get

$$
\{F \circ \chi, G\}=\left\{F, G \circ \chi^{-1}\right\} \circ \chi(U) .
$$

The Birkhoff method relies on the construction of the symplectic diffeomorphism $\chi$ from an auxiliary given function $F, C^{1}$ on an open subset of $H^{s}\left(X, \mathbb{R}^{2}\right) \simeq H^{s}(X, \mathbb{C})$, such that $U \rightarrow X_{F}(U)$ is also $C^{1}$. Actually, the Cauchy-Lipschitz theorem allows one to solve the ODE

$$
\begin{aligned}
\frac{\partial \Phi}{\partial \sigma}(\sigma, U) & =X_{F}(\Phi(\sigma, U)) \\
\Phi(0, U) & =U
\end{aligned}
$$


and for small enough initial data $U$, the solution is defined for $\sigma$ in $[-1,1]$. If one sets

$$
\chi(U)=\Phi(-1, U)
$$

then $\chi$ is symplectic by construction. We may then state:

Lemma 8 Let $G$ be given as in (5.12), (5.13). Assume that one may find a polynomial function $F(U)=\sum_{n \geq 1} F_{n}(U)$, where $F_{n}$ is homogeneous of order $n+2$, such that, if we define

$$
\operatorname{Ad}_{F} G=\{F, G\}, \operatorname{Ad}_{F}^{k} G=\operatorname{Ad}_{F} \operatorname{Ad}_{F}^{k-1} G, k \geq 1,
$$

then

$$
\left\{\Theta_{s}^{0}, \sum_{k=0}^{N-1} \frac{1}{k !} \operatorname{Ad}_{F}^{k} G\right\}=O\left(\|U\|_{H^{s}}^{N+2}\right), U \rightarrow 0 .
$$

Then Theorem 7 holds.

Proof: This is just the basic computation of the Birkhoff normal forms method. If $U$ is a solution to (5.21) and $\Theta_{s}$ is defined by (5.17), with $\chi$ given by (5.22), one has by $(5.18)$ and $(5.20)$

$$
\begin{aligned}
\frac{d}{d t} \Theta_{s}(U(t, \cdot)) & =D \Theta_{s} U(t, \cdot) \cdot \frac{d U}{d t}=D \Theta_{s}(U) \cdot X_{G}(U) \\
& =-\left\{\Theta_{s}, G\right\}=-\left\{\Theta_{s}^{0} \circ \chi, G\right\}=-\left\{\Theta_{s}^{0}, G \circ \chi^{-1}\right\} \circ \chi .
\end{aligned}
$$

In order to prove the theorem, we just have to check that, if $F$ satisfies (5.24), then (5.16) holds ((5.15) will follow from the fact that $\chi$ is a local diffeomorphism with $\chi(0)=0)$. By the definition (5.22) of $\chi$ and Taylor formula,

$$
\begin{aligned}
G \circ \chi^{-1}(U) & =G \circ \Phi(1, U) \\
& =\left.\sum_{k=0}^{N-1} \frac{1}{k !} \frac{d^{k}}{d \sigma^{k}}(G \circ \Phi(\sigma, \cdot))\right|_{\sigma=0}+O\left(\|U\|_{H^{s}}^{N+2}\right) .
\end{aligned}
$$

But, according to (5.21), (5.18),

$$
\frac{d}{d \sigma} G \circ \Phi(\sigma, \cdot)=D G \cdot \frac{d \Phi}{d \sigma}=D G \cdot X_{F}(\Phi)=\{F, G\}(\Phi(\sigma, \cdot))
$$

and more generally, $\frac{d^{k}}{d \sigma^{k}} G \circ \Phi(\sigma, \cdot)=\operatorname{Ad}_{F}^{k} G(\Phi(\sigma, \cdot))$. We thus get from (5.26)

$$
G \circ \chi^{-1}(U)=\sum_{k=0}^{N-1} \frac{1}{k !} \operatorname{Ad}_{F}^{k} \cdot G(U)+O\left(\|U\|_{H^{s}}^{N+2}\right) .
$$

Plugging this equality in (5.25) and using assumption (5.24), we do get, since $\chi(0)=$ 0 , that $\frac{d}{d t} \Theta_{s}(U(t, \cdot))=O\left(\|U\|_{H^{s}}^{N+2}\right)$ i.e. property (5.16). This concludes the proof. 


\section{Resolution of the homological equation}

In lemma 8 of section 5 , we have reduced the proof of Theorem 7 to the resolution of the homological equation (5.24). The goal of this section is to perform this last step, i.e. to prove the following proposition.

Proposition 9 Let $G_{0}(U)=\int_{X}\left(\Lambda_{m} u\right) \bar{u} d x$ and for $n \geq 1$ define

$$
G_{n}(U)=\sum_{p=0}^{n+2} \int_{X} P_{n, p}(u, \ldots, u, \bar{u}, \ldots, \bar{u}),
$$

where $P_{n, p}$ is defined after (5.13). Then for any $N$, there is a polynomial $F(U)=$ $\sum_{n \geq 1} F_{n}(U)$, with $F_{n}$ homogeneous of degree $n+2$, such that the homological equation

$$
\left\{\Theta_{s}^{0}, \sum_{k=0}^{N-1} \frac{1}{k !} \operatorname{Ad}_{F}^{k} G\right\}=O\left(\|U\|_{H^{s}}^{N+2}\right), U \rightarrow 0
$$

holds.

We shall make clear in the proof what we mean by "polynomial function $F$ ". Actually, the key point will be to define a class of homogeneous functions inside which we may solve (6.2). More precisely, we shall prove the following lemma:

Lemma 10 Assume given for any $n$ in $\mathbb{N}^{*}$, any $\nu>0$, a class $\mathcal{L}_{n}^{\nu}$ of functions homogeneous of degree $n+2$ from $H^{s}$ (with s large enough) to $\mathbb{R}$, such that the following four properties hold true:

(i) For any $n \geq 1$, the function $G_{n}$ defined in (5.13) belongs to $\mathcal{L}_{n}^{\nu}$ for some $\nu$.

(ii) For any $\nu, n, n^{\prime}$, there is $\nu^{\prime}$ such that $\left\{\mathcal{L}_{n}^{\nu}, \mathcal{L}_{n^{\prime}}^{\nu}\right\} \subset \mathcal{L}_{n+n^{\prime}}^{\nu^{\prime}}$

(iii) If $L$ is in $\mathcal{L}_{n}^{\nu}$ and $s$ is large enough, $\left\{\Theta_{s}^{0}, L\right\}(U)=O\left(\|U\|_{H^{s}}^{n+2}\right)$.

(iv) If $H_{n}$ is in $\mathcal{L}_{n}^{\nu}$, there are some $\nu^{\prime}$ and $F_{n}$ in $\mathcal{L}_{n}^{\nu^{\prime}}$ so that $\left\{F_{n}, G_{0}\right\}$ is in $\mathcal{L}_{n}^{\nu}$ and $\left\{\Theta_{s}^{0},\left\{F_{n}, G_{0}\right\}-H_{n}\right\}=0$.

Then Proposition 9 holds.

Idea of proof of the lemma: According to (5.23), $\operatorname{Ad}_{F}^{k} G$ is a sum of contributions of the form

$$
\left\{F_{n_{1}},\left\{F_{n_{2}},\left\{\cdots\left\{F_{n_{k}}, G_{n_{0}}\right\}\right\}\right\}\right\}
$$

that belong to $\mathcal{L}_{n_{0}+\cdots+n_{k}}^{\nu^{\prime}}$ for some $\nu^{\prime}$. This follows from (i) and the stability property (ii), together with the fact that if $n_{0}=0$, then $\left\{F_{n_{k}}, G_{0}\right\}$ is in $\mathcal{L}_{n_{k}}^{\nu}$ by (iv). As $G=\sum_{n=0}^{N^{\prime}} G_{n}$, we may write the left hand side of (6.2) as

$$
\left\{\Theta_{s}^{0}, \sum_{k=0}^{N-1} \frac{1}{k !} \operatorname{Ad}_{F}^{k} G\right\}=\sum_{n \geq 1}\left\{\Theta_{s}^{0},\left\{F_{n}, G_{0}\right\}-H_{n}\right\},
$$

where the sum in the right hand side is finite and where $H_{n}$ is computed from expressions of the form (6.3) with $n_{0}+\cdots+n_{k}=n$ and $n_{j}<n$ for all $j=1, \ldots, k$, since the only term where one of these $n_{j}$ is equal to $n$ is the $\left\{F_{n}, G_{0}\right\}$ contribution 
in the right hand side of (6.4). By what we said after (6.3), $H_{n}$ is in $\mathcal{L}_{n}^{\nu^{\prime}}$, and since it depends only on $F_{n^{\prime}}$ for $n^{\prime}<n$, we may use (iv) in order to find successively $F_{n}$ solving $\left\{\Theta_{s}^{0},\left\{F_{n}, G_{0}\right\}-H_{n}\right\}=0$ for $n=1, \ldots, N-1$. In that way, the only remaining terms in the right hand side of (6.4) are of the form $\left\{\Theta_{s}^{0}, L\right\}$ for elements $L$ of $\mathcal{L}_{n}^{\nu^{\prime}}$ for some $\nu^{\prime}$ and $n \geq N$. According to (iii), they provide contributions to the right hand side of (6.2). This concludes the proof.

We have thus reduced the proof of Theorem 7 to the construction of classes $\mathcal{L}_{n}^{\nu}$ such that properties (i) to (iv) hold. In order to do so, we define first classes of multilinear maps.

Definition 11 Let $n$ in $\mathbb{N}^{*}$ and $\nu>0$. For $k$ in $\mathbb{N}$, denote by $\Pi_{k}$ the spectral projector associated to the interval $I_{k}$ introduced in Theorem 5. We define $\tilde{\mathcal{L}}_{n}^{\nu}$ as the space of $(n+2)$-linear forms $\left(u_{1}, \ldots, u_{n+2}\right) \rightarrow L\left(u_{1}, \ldots, u_{n+2}\right)$ from $H^{s} \times \cdots \times H^{s}$ to $\mathbb{C}$ such that, for any $N$ in $\mathbb{N}$, there is $C_{N}>0$ and for any $k_{1}, \ldots, k_{n+2}$ in $\mathbb{N}$,

$$
\left|L\left(\Pi_{k_{1}} u_{1}, \ldots, \Pi_{k_{n+2}} u_{n+2}\right)\right| \leq C_{N} \frac{\left(k_{3}^{*}\right)^{\nu+N}}{\left(k_{1}^{*}-k_{2}^{*}+k_{3}^{*}\right)^{N}} \prod_{j=1}^{n+2}\left\|u_{j}\right\|_{L^{2}}
$$

where

$$
\begin{aligned}
k_{1}^{*}=\max \left(k_{1}, \ldots, k_{n+2}\right)+ & \\
& \\
& \geq k_{2}^{*}=\max _{2}\left(k_{1}, \ldots, k_{n+2}\right)+1 \\
& \geq k_{3}^{*}=\max _{3}\left(k_{1}, \ldots, k_{n+2}\right)+1,
\end{aligned}
$$

denoting by $\max _{j}\left(k_{1}, \ldots, k_{n+2}\right)$ the $j$-th largest among all integers $k_{1}, \ldots, k_{n+2}$.

The basic example of an element of $\mathcal{L}_{n}^{\nu}$ is given by

$$
L\left(u_{1}, \ldots, u_{n+2}\right)=\int_{X} u_{1} \cdots u_{n+2} d x
$$

Let us show this in the special case when $X=\mathbb{S}^{d}$, so that each interval $I_{k}$ is reduced to a single point $\left\{\xi_{k}\right\}$. If for instance $k_{1} \geq k_{2} \geq k_{3} \geq \cdots \geq k_{n+2}$, so that $k_{j}^{*}=k_{j}+1$, estimate first the left hand side of (6.5) by

$$
\left|L\left(\Pi_{k_{1}} u_{1}, \ldots, \Pi_{k_{n+2}} u_{n+2}\right)\right| \leq\left\|\Pi_{k_{1}} u_{1}\right\|_{L^{2}}\left\|\Pi_{k_{2}} u_{2}\right\|_{L^{2}} \prod_{j=3}^{n+2}\left\|\Pi_{k_{j}} u_{j}\right\|_{L^{\infty}}
$$

and use Sobolev injection to estimate the $L^{\infty}$ norms by

$$
\prod_{j=3}^{n+2}\left\|\Pi_{k_{j}} u_{j}\right\|_{L^{\infty}} \leq\left(k_{3}^{*}\right)^{\nu} \prod_{j=3}^{n+2}\left\|\Pi_{k_{j}} u_{j}\right\|_{L^{2}}
$$

for some $\nu$. We thus obtain (6.5) in the special case $k_{1}^{*}-k_{2}^{*} \leq C k_{3}^{*}$. On the other hand, if $k_{1}^{*}-k_{2}^{*} \gg k_{3}^{*}$, we may write, setting $a(x)=\prod_{j=3}^{n+2} \Pi_{k_{j}} u_{j}$, 


$$
\begin{aligned}
&\left(\xi_{k_{1}}^{2}-\xi_{k_{2}}^{2}\right) \int_{X}\left(\Pi_{k_{1}} u_{1}\right)\left(\Pi_{k_{2}} u_{2}\right) a(x) d x \\
&=\int\left[-\left(\Delta \Pi_{k_{1}} u_{1}\right)\left(\Pi_{k_{2}} u_{2}\right)+\left(\Pi_{k_{1}} u_{1}\right)\left(\Delta \Pi_{k_{2}} u_{2}\right)\right] a(x) d x \\
& \quad=\int_{X}\left(\Pi_{k_{1}} u_{2}\right)[a, \Delta]\left(\Pi_{k_{2}} u_{2}\right) d x .
\end{aligned}
$$

It follows, as the $\mathcal{L}\left(H^{1}, L^{2}\right)$ norm of the commutator $[a, \Delta]$ may be bounded from $\|\nabla a\|_{L^{\infty}}$, that

$$
\left(\xi_{k_{1}}^{2}-\xi_{k_{2}}^{2}\right)\left|L\left(\Pi_{k_{1}} u_{1}, \ldots, \Pi_{k_{n+2}} u_{n+2}\right)\right| \leq C\left(k_{3}^{*}\right)^{\nu+1} k_{2}^{*} \prod_{j=3}^{n+2}\left\|\Pi_{k_{j}} u_{j}\right\|_{L^{2}} .
$$

As $\xi_{k_{j}} \sim k_{j}$, this implies (6.5) with $N=1$. Iterating the process, one gets the result.

\section{Remarks}

- We gave the proof of estimate (6.5) for the example (6.6) in the case when $X$ is the sphere. Actually, the above proof may be adapted to an arbitrary compact Riemannian manifold (with the $\Pi_{k_{j}}$ defined from the intervals $I_{k_{j}}$ of Proposition 2). In other words, we do not use any geometric assumption in the proof of the fact that (6.6) belongs to the class $\tilde{\mathcal{L}}_{n}^{\nu}$.

- The same proof shows that $\int_{X} \prod_{j=1}^{n+2}\left(\Lambda_{m}^{-\frac{1}{2}} u_{j}\right) d x$ belongs to $\tilde{\mathcal{L}}_{n}^{\nu}$.

Let us define now the classes $\mathcal{L}_{n}^{\nu}$ that will enjoy the properties of lemma 10.

Definition 12 One says that $U \rightarrow H_{n}(U)$ is in $\mathcal{L}_{n}^{\nu}$ if there are elements $H_{n, p}$ of $\tilde{\mathcal{L}}_{n}^{\nu}$ for $p=0, \ldots, n+2$ such that

$$
H_{n}(U)=\sum_{p=0}^{n+2} H_{n, p}(\underbrace{u, \ldots, u}_{p}, \underbrace{\bar{u}, \ldots, \bar{u}}_{n+2-p}) .
$$

We have to check that properties (i) to (iv) of lemma 10 hold true.

- Property (i) follows from the above example. Actually, by (5.8), $G_{n}$ may be written as an expression (6.7), where $H_{n, p}$ is given by a quantity of the form (6.6) with each factor $u_{j}$ replaced by $\Lambda_{m}^{-\frac{1}{2}} u_{j}$, that provides an element of $\mathcal{L}_{n}^{\nu}$ as already remarked.

- Property (ii), the stability under Poisson brackets, may be reduced to the proof of the fact that if $L$ is in $\tilde{\mathcal{L}}_{n}^{\nu}$ and if $m$ is a $\left(n^{\prime}+1\right)$-linear map $\left(u_{1}, \ldots, u_{n^{\prime}+1}\right) \rightarrow$ $M\left(u_{1}, \ldots, u_{n^{\prime}+1}\right)$, satisfying estimates of the form

$$
\left\|\Pi_{k_{n^{\prime}+2}} M\left(\Pi_{k_{1}} u_{1}, \ldots, \Pi_{k_{n^{\prime}+1}} u_{n^{\prime}+1}\right)\right\|_{L^{2}} \leq C_{N} \frac{\left(k_{3}^{*}\right)^{\nu+N}}{\left(k_{1}^{*}-k_{2}^{*}+k_{3}^{*}\right)^{N}} \prod_{j=1}^{n^{\prime}+1}\left\|u_{j}\right\|_{L^{2}}
$$

then

$$
L\left(M\left(u_{1}, \ldots, u_{n^{\prime}+1}\right), u_{n^{\prime}+2}, \ldots, u_{n+n^{\prime}+2}\right)
$$


is in $\tilde{\mathcal{L}}_{n+n^{\prime}}^{\nu^{\prime}}$ for some $\nu^{\prime}$. This follows from the combination of estimates (6.5) and (6.8) and some elementary computations.

- Property (iii) may be deduced as well from the bounds (6.5) if one takes $s$ large enough relatively to $\nu$.

Finally, we have reduced the proof of Theorem 7 to the fact that we may solve the homological equation of (iv) of lemme 10 i.e. prove the following proposition.

Proposition 13 There is a zero measure subset $\mathcal{N}$ of $] 0,+\infty[$ such that, if $m$ belongs to $] 0,+\infty\left[\right.$, for any $\nu>0, n \in \mathbb{N}^{*}$, any $H_{n}$ in $\mathcal{L}_{n}^{\nu}$, one may find $F_{n}$ in $\mathcal{L}_{n}^{\nu^{\prime}}$, with some $\nu^{\prime}$ depending only on $\nu, m$, such that

$$
\left\{\Theta_{s}^{0},\left\{F_{n}, G_{0}\right\}-H_{n}\right\}=0 .
$$

We shall proceed as in section 3, except that we make the reasoning on linear forms like $H_{n}, F_{n}$ instead of linear maps, in order to exploit the Hamiltonian structure. We prove first the proposition in the simpler case of odd $n$, where we may actually solve a stronger equation than (6.9).

Lemma 14 Assume that $n$ is odd. Then, with the notation of Proposition 13, we may find for any $H_{n}$ in $\mathcal{L}_{n}^{\nu}$ some $F_{n}$ in $\mathcal{L}_{n}^{\nu^{\prime}}$ solving

$$
\left\{F_{n}, G_{0}\right\}-H_{n}=0 \text {. }
$$

Proof: We give the proof in the special case $X=\mathbb{S}^{d}$ so that each interval $I_{k}$ is reduced to the point $\left\{\xi_{k}\right\}$. By definition 12, we may write $H_{n}$ under the form (6.7), and we look for $F_{n}$ as $F_{n}(U)=\sum_{p=0}^{n+2} F_{n, p}(u, \ldots, u, \bar{u}, \ldots, \bar{u})$ with $F_{n, p}$ in $\tilde{\mathcal{L}}_{n}^{\nu^{\prime}}$. To solve (6.10) we have to find $F_{n, p}$ so that

$$
\left\{F_{n, p}(u, \ldots, u, \bar{u}, \ldots, \bar{u}), G_{0}\right\}=H_{n, p}(u, \ldots, u, \bar{u}, \ldots, \bar{u}) .
$$

Recall from (5.19) that

$$
\left\{F_{n, p}, G_{0}\right\}=-i\left[D_{u} F_{n, p} \nabla_{\bar{u}} G_{0}-D_{\bar{u}} F_{n, p} \nabla_{u} G_{0}\right]
$$

so that, since by (5.13) $\nabla_{\bar{u}} G_{0}=\Lambda_{m} u, \nabla_{u} G_{0}=\Lambda_{m} \bar{u}$, equality (6.11) will follow from

$$
\begin{array}{r}
\sum_{j=1}^{p} F_{n, p}\left(u_{1}, \ldots, \Lambda_{m} u_{j}, \ldots, u_{n+2}\right)-\sum_{j=p+1}^{n+2} F_{n, p}\left(u_{1}, \ldots, \Lambda_{m} u_{j}, \ldots, u_{n+2}\right) \\
=i H_{n, p}\left(u_{1}, \ldots, u_{n+2}\right) .
\end{array}
$$

We replace in (6.12) $u_{j}$ by $\Pi_{k_{j}} u_{j}$. Since we assume that $I_{k_{j}}=\left\{\xi_{k_{j}}\right\}$, we have $\Lambda_{m} \Pi_{k_{j}} u_{j}=\sqrt{m^{2}+\xi_{k_{j}}^{2}} \Pi_{k_{j}} u_{j}$, so that (6.12) may be written as

$$
\mathcal{G}_{m}^{p}\left(\xi_{k_{1}}, \ldots, \xi_{k_{n+2}}\right) F_{n, p}\left(\Pi_{k_{1}} u_{1}, \ldots, \Pi_{k_{n+2}} u_{n+2}\right)=i H_{n, p}\left(\Pi_{k_{1}} u_{1}, \ldots, \Pi_{k_{n+2}} u_{n+2}\right)
$$

using notation (3.6). If $n$ is odd, assumption (4.3) of Proposition 6 holds, so that by (4.2) we may divide $(6.13)$ by $\mathcal{G}_{m}^{p}\left(\xi_{k_{1}}, \ldots, \xi_{k_{n+2}}\right)$ and get that if $H_{n, p}$ satisfies bounds of the form (6.5), so does $F_{m, p}$ with $\nu$ replaced by $\nu^{\prime}=\nu+N_{0}$. 
This concludes the proof when $X=\mathbb{S}^{d}$. When $X$ is a general Zoll manifold, each interval $I_{k}$ contains several eigenvalues. But, since the length of $I_{k}$ is $O\left(k^{-\delta}\right)$, they are close enough to each other so that one may still solve (6.13) by a perturbative argument (see $[12,2])$.

End of proof of Proposition 13: We have to complement the conclusion of the preceding lemma treating the case of even $n$, for which condition (4.3) is not always satisfied. Actually, it still holds except if $n$ is even, $p=\frac{n}{2}+1$ and $\left\{k_{1}, \ldots, k_{p}\right\}=$ $\left\{k_{p+1}, \ldots, k_{n+2}\right\}$, in which case $\mathcal{G}_{m}^{p}$ vanishes identically. But notice that we have to solve (6.9) and not (6.10), and by definition of $\Theta_{s}^{0}$, if $\left\{k_{1}, \ldots, k_{p}\right\}=\left\{k_{p+1}, \ldots, k_{n+2}\right\}$,

$$
\begin{aligned}
& \left\{\Theta_{s}^{0}, H_{n, p}\left(\Pi_{k_{1}} u_{1}, \ldots, \Pi_{k_{p}} u_{p}, \Pi_{k_{1}} \bar{u}_{1}, \ldots, \Pi_{k_{p}} \bar{u}_{p}\right)\right\} \\
& \quad=-i\left(\sum_{j=1}^{p}\left\langle\xi_{k_{j}}\right\rangle^{2 s}-\sum_{j=p+1}^{n+2}\left\langle\xi_{k_{j}}\right\rangle^{2 s}\right) H_{n, p}\left(\Pi_{k_{1}} u_{1}, \ldots, \Pi_{k_{p}} u_{p}, \Pi_{k_{1}} \bar{u}_{1}, \ldots, \Pi_{k_{p}} \bar{u}_{p}\right)=0
\end{aligned}
$$

so that, for these values of the indices, we may just define

$$
F_{n, p}\left(\Pi_{k_{1}} u_{1}, \ldots, \Pi_{k_{n+2}} u_{n+2}\right) \equiv 0 .
$$

This concludes the proof.

\section{Quasi-linear equations}

Up to now, we have discussed long time existence problems for semi-linear equations of the form

$$
\left(\partial_{t}^{2}-\Delta+m^{2}\right) w=N(w)
$$

where the non-linearity $N(w)$ vanishes at least at order two at zero and does not depend on derivatives of $w$. We would like now to discuss non-linearities depending also on derivatives of $w$. We cannot hope for a statement as theorem 7, even on $X=\mathbb{S}^{1}$, for any semi-linear non-linearity depending on derivatives of $u$. Actually, examples of Yordanov [23] and Keel and Tao [17] show that, in general, one cannot expect existence over a time interval of length larger than the one given by local existence theory. This shows that, if in the right hand side of $(7.1) N(w)$ is replaced by an expression containing first order derivatives of $w$, the normal form procedure we used in the preceding sections will not always work. The key point is that some contributions to the non-linearity that may not be eliminated by normal forms will induce a growth of the Sobolev energy. On the contrary, if the non-linearity depends only on $w$, the Hamiltonian formulation that we introduced in section 5 showed that all non eliminable contributions do not make grow the energy, as they depend only on the actions, so that, in an homological equation of the form (6.9), their Poisson bracket with $\Theta_{s}^{0}$ vanishes.

If we want to study non-linearities depending also on derivatives, we thus have to restrict ourselves to classes of nonlinear expressions that possess some special properties that ensure that non eliminable terms in the normal form procedure do 
not make grow energy. Moreover, this special structure has to be preserved under normal forms reduction, so that one is able to take profit of it at any step of the construction.

One possibility in order to achieve that is to consider "reversible" equations. We refer to the paper [13] of Fang, Han and Zhang, devoted to semi-linear equations on the circle, with non-linearities depending on a reversible way of $\partial_{t} w, \partial_{x} w$, i.e. verifying the condition

$$
N\left(-w, \partial_{t} w,-\partial_{x} w\right)=-N\left(w, \partial_{t} w, \partial_{x} w\right) .
$$

For odd or even small initial data, they get almost global solutions, when the mass parameter stays outside some subset of zero measure.

Another option, that we shall describe below, is to stay in the Hamiltonian framework, but allowing the Hamiltonian (5.5) to depend not only on $w$, but also on $\partial_{x} w$, so that, in $(5.8), G(V)$ would be given in terms of an integral of $P\left(\Lambda_{m}^{\frac{1}{2}} V\right)$ instead of $P\left(\Lambda_{m}^{-\frac{1}{2}} V\right)$. In this case, the evolution equation is necessarily a quasi-linear one, and not just semi-linear. In fact, one may check that if a non-linearity is both semi-linear and Hamiltonian, then it has to depend only on $w$ and not its derivatives, so that one gets just equation (7.1). In this section, we shall thus state a theorem of almost global existence for quasi-linear Hamiltonian Klein-Gordon equations on spheres.

Let us fix some notation. From now on, $X$ will stand for the sphere $\mathbb{S}^{d}$. Let $f: \mathbb{R} \times T^{*} X \rightarrow \mathbb{R}$ be a smooth map $(z, \rho) \rightarrow f(z, \rho)$, polynomial in the fiber variable of $\rho$, and vanishing at least at order 3 at $(z=0, \rho \in X)$, where we identify $X$ to the zero section of $T^{*} X$. We consider a non-linearity $P[w]$ given in local coordinates by

$$
P[w]=\frac{\partial f}{\partial z}\left(w, x, \frac{\partial w}{\partial x}\right)-\sum_{j=1}^{d}(\operatorname{det} g)^{-1 / 2} \frac{\partial}{\partial x_{j}}\left[(\operatorname{det} g)^{1 / 2}\left(\frac{\partial f}{\partial \xi_{j}}\right)\left(w, x, \frac{\partial w}{\partial x}\right)\right]
$$

(where $g$ is the metric of the sphere) and the quasi-linear Klein-Gordon equation

$$
\left(\partial_{t}^{2}-\Delta+m^{2}\right) w=P[w] .
$$

This is a Hamiltonian equation associated to the Hamiltonian

$$
\int_{X}\left[\frac{1}{2}\left[\left(\partial_{t} w\right)^{2}+\left(\partial_{x} w\right)^{2}+m^{2} w^{2}\right]-f(w, d w)\right] d x
$$

If we set

$$
F(w)=\int_{X} f(w, d w) d x
$$

and define $V$ from $\left(w, \partial_{t} w\right)$ as in (5.4), equation (7.3) may be rewritten as

$$
\partial_{t} V=X_{G}(V)
$$

with

$$
G(V)=G_{0}(V)-F\left(\Lambda_{m}^{-\frac{1}{2}} V^{2}\right), G_{0}(V)=\frac{1}{2} \int_{X}\left(\Lambda_{m}^{\frac{1}{2}} V\right) \cdot\left(\Lambda_{m}^{\frac{1}{2}} V\right) d x
$$


where, because of the first order derivative in $(7.5), F\left(\Lambda_{m}^{-\frac{1}{2}} V^{2}\right)$ is actually of order $\frac{1}{2}$ in $V^{2}$. Defining as before $u=\frac{\sqrt{2}}{2}\left(V^{1}+i V^{2}\right), U=(u, \bar{u})$ and writing the expression of the Hamiltonian in terms of $U$, namely

$$
G(U)=\int_{X}\left(\Lambda_{m} u\right) \bar{u} d x-F\left(-i \frac{\sqrt{2}}{2} \Lambda_{m}^{-\frac{1}{2}}(u-\bar{u})\right)
$$

the equation may be written again

$$
\begin{aligned}
\dot{u} & =i \nabla_{\bar{u}} G(U) \\
\left.u\right|_{t=0} & =\epsilon u_{0} .
\end{aligned}
$$

The new feature is that $F$ in (7.8) being of the form (7.5), one should think of $G(U)$ as $\sum_{n=0}^{N^{\prime}} G_{n}(U)$, with $G_{0}(U)$ given by the first formula in (5.13), but where $G_{n}(U)$ is no longer given by the second formula (5.13), but instead by an expression of the form

$$
G_{n}(U)=\sum_{p=0}^{n+2} \int_{X} P_{n, p}\left(\Lambda_{m}^{\frac{1}{2}} u, \ldots, \Lambda_{m}^{\frac{1}{2}} u, \Lambda_{m}^{\frac{1}{2}} \bar{u}, \ldots, \Lambda_{m}^{\frac{1}{2}} \bar{u}\right)
$$

with $P_{n, p}$ a $(n+2)$-linear form. Actually, the expression one gets is more general than (7.10), since it involves operators of order $\frac{1}{2}$ that are not necessarily the $\Lambda_{m}^{\frac{1}{2}}$ 's.

The main theorem is as follows:

Theorem 15 With the above notation, there is a zero measure subset $\mathcal{N}$ of $] 0,+\infty[$ and for any $m \in] 0,+\infty\left[-\mathcal{N}\right.$, any $N \in \mathbb{N}$, there is $s_{0}>0$ and for any $s>s_{0}$, there are $\epsilon_{0}>0, c>0, C>0$, such that, for any $\left.\epsilon \in\right] 0, \epsilon_{0}\left[\right.$, any $\left(w_{0}, w_{1}\right)$ in the unit ball of $H^{s+\frac{1}{2}}(X) \times H^{s-\frac{1}{2}}(X)$, one may find a unique $w$ in

$$
C^{0}(]-T_{\epsilon}, T_{\epsilon}\left[, H^{s+\frac{1}{2}}(X)\right) \cap C^{1}(]-T_{\epsilon}, T_{\epsilon}\left[, H^{s-\frac{1}{2}}(X)\right)
$$

solution of

$$
\begin{aligned}
\left(\partial_{t}^{2}-\Delta+m^{2}\right) w & =P[w] \\
\left.w\right|_{t=0} & =\epsilon w_{0} \\
\left.\partial_{t} w\right|_{t=0} & =\epsilon w_{1},
\end{aligned}
$$

where $T_{\epsilon} \geq c \epsilon^{-N}$ and

$$
\sup _{]-T_{\epsilon}, T_{\epsilon}[}\left[\|w(t, \cdot)\|_{H^{s+\frac{1}{2}}}+\left\|\partial_{t} w(t, \cdot)\right\|_{H^{s-\frac{1}{2}}}\right] \leq C \epsilon .
$$

This theorem is proved in [10] (see also [9] for the case $X=\mathbb{S}^{1}$ ). We shall discuss some ideas of the proof in the model case (that already contains all difficulties) given by (7.9) with $G(U)=\sum_{n=0}^{N^{\prime}} G_{n}(U)$, where $G_{0}(U)=\int_{X}\left(\Lambda_{m} u\right) \bar{u} d x$ and $G_{n}$ given by (7.10) for $n \geq 1$.

Let us explain the main new ideas that have to be introduced. In the semilinear case, we have instead of (7.10) an expression of $G_{n}$ given by (5.13) i.e. by generalization of quantities of the form

$$
\int_{X} u \cdots u \bar{u} \cdots \bar{u} d x .
$$


We may write (7.13) in terms of expressions

$$
\int_{X}(A(U) u) \bar{u} d x, \int_{X}(A(U) u) u d x, \int_{X}(A(U) \bar{u}) \bar{u} d x
$$

where $A(U)$ is a quantity of the from $u^{n^{\prime}} \bar{u}^{n^{\prime \prime}}$ with $n^{\prime}+n^{\prime \prime}=n$. The operator $A(U)$ is thus of order zero, so that it does not lose any derivative. In the homological equation (6.9), we could have written the function $H_{n}$ under the form (7.14) as well. The solution $F_{n}$ we have constructed in the proof of Proposition 13 has the same form as $H_{n}$. Moreover, the contributions to $H_{n+1}, H_{n+2}, \ldots$ generated by $F_{n}$ through Poisson brackets in (6.3) have also structure (7.14). Actually, the Poisson bracket between two functions of the form (7.14) involving operators $A^{\prime}(U), A^{\prime \prime}(U)$ may be written as (7.14) with $A(U)$ replaced by $A^{\prime}(U) \circ A^{\prime \prime}(U)$ or $A^{\prime \prime}(U) \circ A^{\prime}(U)$. As $A^{\prime}, A^{\prime \prime}$ are of order zero, so is $A$ i.e. the structure (7.14) is preserved along the normal forms procedure.

For quasi-linear equations, this is no longer the case at first glance. If instead of (7.13) we start from a formula similar to (7.10), namely

$$
\int_{X}\left(\Lambda_{m}^{\frac{1}{2}} u\right) \cdots\left(\Lambda_{m}^{\frac{1}{2}} u\right)\left(\Lambda_{m}^{\frac{1}{2}} \bar{u}\right) \cdots\left(\Lambda_{m}^{\frac{1}{2}} \bar{u}\right) d x
$$

we get in (7.14) operators of order one. Then the functions $F_{n}$ obtained solving the homological equation are expected to be also given by (7.14), with operators $A(U)$ of order one, so that the contributions induced by Poisson bracket between $F_{n}$ and $H_{n^{\prime}}$ are expected to contain compositions of operators of order one, that is operators of order two. Clearly, each new step would generate operators of higher and higher order, so that the procedure would lead to derivative losses.

In order to overcome these difficulties, one has to show that the structure of the Poisson bracket allows one to avoid these increasing losses. Actually, one may check easily that when computing

$$
\left\{\int_{X}\left(A^{\prime}(U) u\right) \bar{u} d x, \int_{X}\left(A^{\prime \prime}(U) u\right) \bar{u} d x\right\}
$$

one gets a main contribution of the form

$$
\int_{X}\left(\left[A^{\prime}(U), A^{\prime \prime}(U)\right] u\right) \bar{u} d x
$$

plus other terms having still the structure (7.14) with $A(U)$ of order one. We thus need to know that $\left[A^{\prime}(U), A^{\prime \prime}(U)\right]$ is also of order one, so that we stay in the same classes of integral quantities. In other words, we need to know that $A^{\prime}(U), A^{\prime \prime}(U)$ belong to a class of operators enjoying some "symbolic calculus". Examples of such classes are pseudo-differential operators. Since in our problem, the coefficients of the operators depend on the unknown $U$, that has limited regularity, it is actually better to use para-differential operators. We shall not recall here the definition of the latter. Let us just say that one may define for any $m$ in $\mathbb{R}, n$ in $\mathbb{N}$, families of operators $H \Psi_{n}^{\ell}$, that are homogeneous polynomials of degree $n$ in $U$, such that for any fixed $U$, the operator $V \rightarrow A(U) V$ satisfies the following: 
- If $A$ is in $H \Psi_{n}^{\ell}$, then for any $s$ in $\mathbb{R}, V \rightarrow A(U) V$ sends $H^{s}(X)$ to $H^{s-\ell}(X)$ (and its operator norm depends only on $\|U\|_{H^{\sigma}}$ for some $\sigma$ independent of $s$ ).

- If $A$ is in $H \Psi_{n}^{\ell}$ and $B$ is in $H \Psi_{n^{\prime}}^{\ell^{\prime}}$, then $A \circ B$ is in $H \Psi_{n+n^{\prime}}^{\ell+\ell^{\prime}}$ and $[A, B]$ is in $H \Psi_{n+n^{\prime}}^{\ell+\ell^{\prime}-1}$.

One may show that equation (7.11) may be written equivalently as

$$
\begin{aligned}
\dot{u} & =i \nabla_{\bar{u}} G(U) \\
\left.u\right|_{t=0} & =\epsilon u_{0}
\end{aligned}
$$

where $G(U)=\sum_{n=0}^{N^{\prime}} G_{n}(U)$ with essentially

$$
G_{n}(U)=\operatorname{Re} \int_{X}\left(A_{n}^{1}(U) u\right) \bar{u} d x+\operatorname{Re} \int_{X}\left(A_{n}^{2}(U) u\right) u d x
$$

with $A_{n}^{1}, A_{n}^{2}$ in $H \Psi_{n}^{1}$. Let us consider the model case $A_{n}^{2}=0$ and prove:

Proposition 16 Let $A_{n}$ be in $H \Psi_{n}^{1}$ for $n=1, \ldots, N^{\prime}$ and define for $n \geq 1, G_{n}(U)=$ $\int_{X}\left(A_{n}(U) u\right) \bar{u} d x$ and $G(U)=\sum_{n=0}^{N^{\prime}} G_{n}(U)$. Then, for any $N$ in $\mathbb{N}$, if $m$ is outside a subset $\mathcal{N}$ of zero measure of $] 0,+\infty\left[\right.$, there is $s_{0}>0$ and for any $s>s_{0}$, there are $\epsilon_{0}>0, c>0, C>0$ such that, if $\left.\epsilon \in\right] 0, \epsilon_{0}\left[, u_{0}\right.$ is in the unit ball of $H^{s}(X, \mathbb{C})$, system (7.18) has a unique solution $u$ in $C^{0}(]-T_{\epsilon}, T_{\epsilon}\left[, H^{s}(X)\right)$ with $T_{\epsilon} \geq c \epsilon^{-N}$ and $\sup _{]-T_{\epsilon}, T_{\epsilon}}\|u(t, \cdot)\|_{H^{s}} \leq C \epsilon$.

Strategy of proof: The strategy of proof is similar to the one we used in the semilinear case, except that we have to exploit the expression of the $G_{n}$ in terms of para-differential operators in order to avoid derivative losses.

\section{First step: Construction of modified energy}

We have seen that the first step in the proof of long time existence is the construction of a modified energy $\Theta_{s}$ of the form (5.17). In the semi-linear case, $\Theta_{s}$ was defined using the canonical transformation $\chi$ given by (5.22) from the solution $\Phi$ of the ODE (5.21). In the quasi-linear framework, (5.21) is no longer an ODE, as the right hand side $X_{F}$ will have to be defined in terms of a function $F$ given by

$$
\int_{X}(B(U) u) \bar{u} d x
$$

with $B(U)$ of order one. Thus, (5.21) is then a PDE involving in its right hand side the action of the operator of order one $B(U)$. Though, it would be still possible to solve the corresponding evolution equation for $\Phi$, as the PDE one gets is an hyperbolic one. Nevertheless, it is not even necessary to do so, because one just needs a finite Taylor expansion of $G \circ \chi^{-1}$ (see (5.26)), so that it is enough to define the action of composition of $G$ with $\chi^{-1}$ at finite order, i.e. to give a meaning to the sum in the right hand side of (5.27). The general term of that sum is made of Poisson brackets like

$$
\left\{\int_{X}\left(B_{n^{\prime}}(U) u\right) \bar{u} d x, \int_{X}\left(A_{n^{\prime \prime}}(U) u\right) \bar{u} d x\right\}
$$

with $A_{n^{\prime \prime}}, B_{n^{\prime}}$ para-differential operators of order one. According to (7.17) and to the fact that commutators $\left[B_{n^{\prime}}, A_{n^{\prime \prime}}\right]$ are still in $H \Psi_{n^{\prime}+n^{\prime \prime}}^{1}$, one sees that the sum in 
(5.27) will be made essentially of terms of the form (7.20) with operators $B(U)$ of order one.

\section{Second step: Resolution of the homological equation}

As in the semi-linear case, the first step reduces the proof of Proposition 16 to an homological equation of the form (6.9). For the sake of simplicity, let us consider only odd degrees of homogeneity $n$, so that one can solve instead of (6.9)

$$
\left\{F_{n}, G_{0}\right\}-H_{n}=0
$$

where $H_{n}(U)=\int_{X}\left(A_{n}(U) u\right) \bar{u} d x$ for some self-adjoint operator $A_{n}$ in $H \Psi_{n}^{1}$, and look for $F_{n}$ as $\int_{X}\left(B_{n}(U) u\right) \bar{u} d x$, with $B_{n}$ to be determined also in $H \Psi_{n}^{1}$. Looking for $A_{n}, B_{n}$ under the form

$$
A_{n}(U)=\sum_{p=0}^{n} A_{n}^{p}(\underbrace{u, \ldots, u}_{p}, \underbrace{u, \ldots, \bar{u}}_{n-p}), B_{n}(U)=\sum_{p=0}^{n} B_{n}^{p}(\underbrace{u, \ldots, u}_{p}, \underbrace{u, \ldots, \bar{u}}_{n-p})
$$

where $A_{n}^{p}\left(u_{1}, \ldots, u_{n}\right), B_{n}^{p}\left(u_{1}, \ldots, u_{n}\right)$ are again first order para-differential operators that are $n$-linear in $\left(u_{1}, \ldots, u_{n}\right)$, one may rewrite $(7.22)$ as an equation of the form

$$
\begin{array}{r}
{\left[B_{n}^{p}(U), \Lambda_{m}\right]+\sum_{j=1}^{p} B_{n}^{p}\left(u_{1}, \ldots, \Lambda_{m} u_{j}, \ldots, u_{n}\right)-\sum_{j=p+1}^{n} B_{n}^{p}\left(u_{1}, \ldots, \Lambda_{m} u_{j}, \ldots, u_{n}\right)} \\
=-i A_{n}^{p}(\mathcal{U})+\text { remainders }
\end{array}
$$

where $\mathcal{U}=\left(u_{1}, \ldots, u_{n}\right)$. This equation replaces (6.12) in the quasi-linear setting. In order to solve it, one first shows that one may replace in $(7.24)\left[B_{n}^{p}, \Lambda_{m}\right]$ by $\left[B_{n}^{p}, \widetilde{\Lambda}\right]$, where $\widetilde{\Lambda}=\sqrt{-\Delta+\left(\frac{d-1}{2}\right)^{2}}$, up to contributions that are better in terms of the number of derivatives involved in the operator. One is thus essentially reduced to an equation of the form

$$
\begin{array}{r}
{\left[B_{n}^{p}(\mathcal{U}), \widetilde{\Lambda}\right]+\sum_{j=1}^{p} B_{n}^{p}\left(u_{1}, \ldots, \Lambda_{m} u_{j}, \ldots, u_{n}\right)-\sum_{j=p+1}^{n} B_{n}^{p}\left(u_{1}, \ldots, \Lambda_{m} u_{j}, \ldots, u_{n}\right)} \\
=-i A_{n}^{p}(\mathcal{U}) .
\end{array}
$$

One then replaces each $u_{j}$ by $\Pi_{k_{j}} u_{j}$ which, denoting $\Pi_{k} \mathcal{U}=\left(\Pi_{k_{1}} u_{1}, \ldots, \Pi_{k_{n}} u_{n}\right)$, brings (7.25) to

$$
\left[B_{n}^{p}\left(\Pi_{k} \mathcal{U}\right), \widetilde{\Lambda}\right]+\tilde{\mathcal{G}}_{m}^{p}\left(\xi_{k_{1}}, \ldots, \xi_{k_{n}}\right) B_{n}^{p}\left(\Pi_{k} \mathcal{U}\right)=-i A_{n}^{p}\left(\Pi_{k} \mathcal{U}\right)
$$

with $\tilde{\mathcal{G}}_{m}^{p}$ given by (4.5), the $\xi_{k_{j}}$ being the eigenvalues of the square root of the laplacian on the sphere. Taking $\theta$ in $C_{0}^{\infty}(\mathbb{R})$, equal to one close to zero, one can check that if we set

$$
B_{n}^{p}\left(\Pi_{k} \mathcal{U}\right)=-\int_{0}^{+\infty} e^{-i t \widetilde{\Lambda}} A\left(\Pi_{k} \mathcal{U}\right) e^{i t \widetilde{\Lambda}} e^{i \tilde{\mathcal{G}}_{m}^{p}\left(\xi_{k_{1}}, \ldots, \xi_{k_{n}}\right)} \theta(\epsilon t) d t
$$


one gets a solution to (7.25), up to a remainder of the form $\epsilon^{N} R$, where $R$ is again a para-differential operator of order one. The contribution of this last term to the homological equation (6.9) is $O\left(\epsilon^{N+2}\right)$, i.e. is $O\left(\|u\|_{H^{s}}^{N+2}\right)$, as the right hand side of (5.16), since $\epsilon$ is essentially the size of $\|u\|_{H^{s}}$.

Finally, the last step of the proof is to show that if $A$ in the right hand side of (7.27) is a para-differential operator of order one, so is $B$. This may be proved using that $t \rightarrow e^{i t \widetilde{\Lambda}}$ is $2 \pi$-periodic (this is the place where one uses that $X$ is a sphere, as this implies that the eigenvalues of $\widetilde{\Lambda}$ are integers $)$ and using that $\tilde{\mathcal{G}}_{m}^{p}\left(\xi_{k_{1}}, \ldots, \xi_{k_{n}}\right)$ satisfies small divisor bounds of the form (3.4). Notice that here, because $A_{n}^{p}\left(\Pi_{k} \mathcal{U}\right)$ is a paradifferential operator, the frequencies $k_{1}, \ldots, k_{n}$ that appear in the argument of $\tilde{\mathcal{G}}_{m}^{p}$ are actually the small frequencies, so that a lower bound in $\left(1+\max \left(k_{1}, \ldots, k_{n}\right)\right)^{-N_{0}}$ is the analogous of the lower bound $(4.2)$ in $\left(1+\max _{3}\left(k_{1}, \ldots, k_{n+2}\right)\right)^{-N_{0}}$. This concludes the sketch of proof of the proposition.

To conclude this section, let us mention that recent results of almost global existence for quasi-linear equations have also been obtained by Berti and Delort in [4] for the capillary-gravity water waves equation on the circle. In this case, the situation is different from the one we considered here, as the linear part of the system is given by an operator of order $\frac{3}{2}$ instead of one. The eigenvalues of that operator are then separated by growing gaps, that allow one to perform a reduction to constant coefficients.

\section{References}

[1] D. Bambusi. Birkhoff normal form for some nonlinear PDEs. Comm. Math. Physics, 234:253-285, 2003.

[2] D. Bambusi, J.-M. Delort, B. Grébert, and J. Szeftel. Almost global existence for Hamiltonian semilinear Klein-Gordon equations with small Cauchy data on Zoll manifolds. Comm. Pure Appl. Math., 60(11):1665-1690, 2007.

[3] D. Bambusi and B. Grébert. Birkhoff normal form for PDEs with tame modulus. Duke Math. J., 135:507-567, 2006.

[4] M. Berti and J.-M. Delort. Almost Global Solutions of Capillary-gravity Water Waves Equations on the Circle. Lecture Notes of the Unione Matematica Italiana. Springer, Cham, To appear, 2018.

[5] A. L. Besse. Manifolds all of whose geodesics are closed, volume 93 of Ergebnisse der Mathematik und ihrer Grenzgebiete. Springer-Verlag, Berlin-New York, 1978. With appendices by D. B. A. Epstein, J.-P. Bourguignon, L. BérardBergery, M. Berger and J. L. Kazdan.

[6] J. Bourgain. Construction of approximative and almost periodic solutions of perturbed linear Schrödinger and wave equations. Geom. Funct. Anal., 6(2):201-230, 1996.

[7] Y. Colin de Verdière. Sur le spectre des opérateurs elliptiques à bicaractéristiques toutes périodiques. Comment. Math. Helv., 54(3):508-522, 1979. 
[8] J.-M. Delort. Temps d'existence pour l'équation de Klein-Gordon semi-linéaire à données petites périodiques. American Journal of Mathematics, pages 663689, 1998.

[9] J.-M. Delort. A quasi-linear Birkhoff normal forms method. Application to the quasi-linear Klein-Gordon equation on $\mathbb{S}^{1}$. Astérisque, (341):vi+113, 2012.

[10] J.-M. Delort. Quasi-linear perturbations of Hamiltonian Klein-Gordon equations on spheres. Mem. Amer. Math. Soc., 234(1103):vi+80, 2015.

[11] J.-M. Delort and R. Imekraz. Long-time existence for the semilinear KleinGordon equation on a compact boundary-less Riemannian manifold. Comm. Partial Differential Equations, 42(3):388-416, 2017.

[12] J.-M. Delort and J. Szeftel. Long-time existence for small data nonlinear KleinGordon equations on tori and spheres. Int. Math. Res. Not., (37):1897-1966, 2004.

[13] D. Fang, Z. Han, and Q. Zhang. Almost global existence for the semi-linear Klein-Gordon equation on the circle. J. Differential Equations, 262(9):46104634, 2017.

[14] D. Fang and Q. Zhang. Long-time existence for semi-linear Klein-Gordon equations on tori. Journal of Differential Equations, 249(1):151-179, 2010.

[15] V. Guillemin. Lectures on spectral theory of elliptic operators. Duke Math. J., 44(3):485-517, 1977.

[16] A. D. Ionescu and F. Pusateri. Long-time existence for multi-dimensional periodic water waves. Preprint, arxiv-1807.02932, 2018.

[17] M. Keel and T. Tao. Small data blow-up for semilinear Klein-Gordon equations. Amer. J. Math., 121(3):629-669, 1999.

[18] S. Klainerman. Global existence of small amplitude solutions to nonlinear KleinGordon equations in four space-time dimensions. Comm. Pure Appl. Math., 38(5):631-641, 1985.

[19] J. Shatah. Normal forms and quadratic nonlinear Klein-Gordon equations. Comm. Pure Appl. Math., 38(5):685-696, 1985.

[20] J. C. H. Simon and E. Taflin. The Cauchy problem for nonlinear Klein-Gordon equations. Comm. Math. Phys., 152(3):433-478, 1993.

[21] A. Stingo. Global existence and asymptotics for quasi-linear one-dimensional Klein-Gordon equations with mildly decaying Cauchy data. Preprint, hal01172523, to appear, Bull. Soc. Math. France.

[22] A. Weinstein. Asymptotics of eigenvalue clusters for the Laplacian plus a potential. Duke Math. J., 44(4):883-892, 1977.

[23] B. Yordanov. Blow-up for the one dimensional Klein-Gordon equation with a cubic nonlinearity. Preprint, 1996. 